\title{
Directed Polymers with Constrained Winding Angle
}

\author{
Franco Ferrari, 1 , * Vakhtang G. Rostiashvili, ${ }^{2,+}$ and Thomas A. Vilgis ${ }^{2, t}$ \\ ${ }^{1}$ Institute of Physics, University of Szczecin, \\ ul. Wielkopolska 15, 70-451 Szczecin, Poland \\ ${ }^{2}$ Max Planck Institute for Polymer Research, \\ 10 Ackermannweg, 55128 Mainz, Germany
}

\begin{abstract}
In this article we study from a non-perturbative point of view the entanglement of two directed polymers subjected to repulsive interactions given by a Dirac $\delta$-function potential. An exact formula of the so-called second moment of the winding angle is derived. This result is used to provide a thorough analysis of entanglement phenomena in the classical system of two polymers subjected to repulsive interactions and related problems. No approximation is made in treating the constraint on the winding angle and the repulsive forces. In particular, we investigate how repulsive forces influence the entanglement degree of the two-polymer system. In the limit of ideal polymers, in which the interactions are switched off, we show that our results are in agreement with those of previous works.
\end{abstract}

\section{INTRODUCTION}

The statistical mechanics of two polymers with constraints on their winding angle has been extensively studied in order to understand the behavior of physical polymer systems, like for instance biological macromolecules of DNA |1| or liquid crystals composed of stacks of disk-shaped molecules [2], see Refs. [3, 44, 5, 66, 7, 18, 9, 10, 11, 12, 13, 14, 15, 16, 17, 18]. A detailed review on the subject, together with interesting proposals of how to include in the treatment of topologically entangled polymer link invariants which are more sophisticated than the winding number, can be found in [19]. Up to now, however, despite many efforts,

*Electronic address: ferrari@univ.szczecin.pl

${ }^{\dagger}$ Electronic address: rostiash@mpip-mainz.mpg.de

${ }^{\ddagger}$ Electronic address: vilgis@mpip-mainz.mpg.de 
mainly ideal polymer chains or loops winding around each others have been considered, while the repulsive interactions between the monomers have been treated approximatively or exploiting in a clever way scaling arguments integrated by numerical simulations, as for instance in [7].

Here we concentrate ourselves on the case of two directed polymers interacting via a repulsive Dirac $\delta$-function potential [20, 21]. We are particularly interested in the average degree of entanglement of the system, which we wish to estimate by computing the square average winding angle of the two polymers. This quantity is also called second moment of the winding angle or simply second moment and is a special example of the topological moments first introduced in Ref. [22]. To achieve our goals, we develop an approach, which combines quantum mechanical and field theoretical techniques. With respect to previous works, we are able to obtain exact results even if the repulsive interactions are not switched off.

In principle, the average of any observable like the squared winding angle can be derived once the partition function of the system is known, but in our case it turns out that the partition function is simply too complicated to obtain any analytical result. This happens essentially because the full $\delta$-function potential is not a central potential, since it mixes both radial and angular variables. For this reason, the usual procedure of going to polar coordinates and then solving the differential equation satisfied by the partition function of the entangled polymers with the method of separation of variables [19], does no longer produce simple formulas as in the situations in which only central forces are present.

To avoid these difficulties, one possibility is to approximate the $\delta$-function potential with some radial potential, like for instance the hard core potential of Ref. [7]. However, here we shall adopt a different strategy, based on field theories, which does not require any approximation. This strategy has been developed in [8, 12] (see also Ref. [19] for more details) to cope with ideal closed polymers whose trajectories are concatenated. Also such systems are characterized by a non-central potential, which comes out as a consequence of the topological constraints imposed on the trajectories. In the field theoretical formulation of Refs. [8, 12, 19] the computation of the second moment is reduced to the problem of computing some correlation functions of a field theory. A bonus is provided by the fact that this computation requires just a finite number of Feynman diagrams to be evaluated. In the present case, due to the presence of the $\delta$-function potential, the field theory which we 
obtain is no longer free as that of Refs. [8, 12, 19]. Nevertheless, we will see that the theory is still linear and thus it can be exactly solved once its propagator is known. Luckily, this propagator may be computed exactly using powerful non-perturbative techniques developed in the context of quantum mechanics to deal with Hamiltonian containing $\delta$-function potentials, see Refs. [23, 24, 25, 26, 27, 28, 29, 30, 31, 32]. Basically, starting from the Green function of a particle whose dynamics is governed by a given Hamiltonian $\mathcal{H}_{0}$, these techniques provide an algorithm to construct the Green function of a particle corresponding to a perturbed Hamiltonian $\mathcal{H}=\mathcal{H}_{0}+V_{\delta}$, where $V_{\delta}$ is the $\delta$-function potential. One advantage of these methods is that there is a long list of potentials for which the Green functions of the unperturbed Hamiltonians $\mathcal{H}_{0}$ are known. In this way, it is easy to generalize our treatment including new interactions, which could be relevant in polymer physics, like for instance the Coulomb interaction. The price to be paid is that the quantum-mechanical algorithm works when the Green functions are expressed as functions of the energy instead of the time. In the polymer analogy, assuming that the ends of the polymers are attached to two planar surfaces perpendicular to the $z$-axis and located at the positions $z=0$ and $z=L$, the role of time is played by the distance $L$, while the energy corresponds to the chemical potential conjugated $L$. To recover the original dependence on $L$, one needs to calculate an inverse Laplace transform of the field propagator with respect to the energy. In general, this is not a simple task.

Once the propagator of the linear field theory is known, the correlation functions which enter in the expression of the second moment may be calculated contracting the fields in all possible ways using the Wick prescription. At the end, we get in this way an exact formula of the second moment as a function of the energy, which, we remember, has here the meaning of the chemical potential conjugated to the distance $L$. In the $L$ space, due to the problems of computing the inverse Laplace transform of the propagator mentioned above, only an approximated expression of the second moment will be given in the limit of large values of $L$ and assuming that the strength of the $\delta$-function potential is weak enough to allow a perturbative approach.

Our results allow both a qualitative and quantitative understanding of the way in which the repulsive interactions affect the entanglement of two directed polymers. The corrections introduced by these interactions in the expression of the second moment of ideal polymers have been studied in some interesting limits. First of all, it has been examined the limit 
of long polymer trajectories, in which we show that repulsive interaction become particularly relevant. Moreover, we have investigated also the perturbative regime and the strong coupling limit, which is important to recover the excluded volume interactions. While it is not a problem to take the strong coupling limit within our exact treatment of the repulsive interactions, it turns out that, in this case, the expression of the second moment is particularly complicated from the analytical point of view. For this reason, in the Conclusions we will discuss the application of a powerful perturbative method to study field theories at strong coupling due to Kleinert [33, 34, 35]. Finally, the consistency of our results with the previous ones has been checked by studying the limit of ideal polymers.

The material presented in this paper is divided as follows. In the next Section, the problem of computing the second moment of the winding angle of two directed polymers interacting via a $\delta$-function potential is briefly illustrated using the path integral approach. A constraint on the winding angle is imposed by coupling the trajectories of the polymers with a suitable external magnetic field, following the strategy of previous works like for instance [8, 14, 15, 19]. In Section [II] the second moment is expressed in the form of a finite sum of amplitudes of a linear field theory. These amplitudes may be computed once the propagator of the theory is constructed. In our case, the propagator coincides with the Green function of a particle diffusing in a $\delta$-function potential. The derivation of this Green function in the energy representation using non-perturbative techniques developed in the context of quantum mechanics [23, 24, 25, 26, 27, 28, 29, 30, 31, 32] is the subject of Section IV The $\delta$-function potential is responsible of the appearance of singularities in the propagator at short distances, which have been regulated here with the introduction of a cut-off. This procedure is motivated by the fact that in polymer physics there is no point in considering distances which are smaller than the dimensions of a monomer. A comparison with the more rigorous method of renormalization is made, showing the consistency of the two procedures. The propagator derived in Section (IV) has a particularly nice form, in which the contributions coming from the repulsive forces can be separated from the free part of the propagator, which is related to the random walk of ideal polymers. This splitting of the propagator is used in Section $\nabla$ to discuss qualitatively and qualitatively the effects of the $\delta$-function interactions on the entanglement of the system. The results of Sections III and IV provide in principle all the ingredients of the second moment. However, the amplitudes of the linear field theory derived in Section III should still be evaluated. In 
this task one encounters the typical problems occurring in the evaluation of the analytical expressions of Feynman diagrams. In the case of the second moment there are just tree diagrams, but still one has to perform complicated integrations over the spatial coordinates which are transverse to the $z$-axis. Even assuming that polymers are ideal, the analytical evaluation of these integrations requires drastic approximations, see for instance [8]. To avoid these difficulties, we average the second moment with respect to the positions of the endpoints of the two polymers. This averaged version of the second moment can be computed without any approximation in the energy representation. This is done in Section VI. The expression of the averaged second moment in the $L$-space is provided instead only at the first perturbative order in the strength of the repulsive potential and assuming additionally that the value of $L$ is large. We give also an exact formula of the second moment without performing any averaging procedure as a function of $L$. This formula is however explicit only up to the calculation of the inverse Laplace transform of the propagator derived in Section IV. In Section VII we consider the situation in which the polymers are not interacting in order to allow the comparison with previous results. Finally, the discussion of the obtained results and ideas for further developments are presented in the Conclusions.

\section{THE STATISTICAL MECHANICS OF TWO DIRECTED POLYMERS WITH CONSTRAINED WINDING ANGLE}

Our starting point is the action of two directed polymers $A$ and $B$ :

$$
\mathcal{A}_{0}=\int_{0}^{L} d z\left[c\left(\frac{d \mathbf{r}_{A}}{d z}\right)^{2}+c\left(\frac{d \mathbf{r}_{B}}{d z}\right)^{2}-V\left(\mathbf{r}_{A}-\mathbf{r}_{B}\right)\right]
$$

where $V\left(\mathbf{r}_{A}-\mathbf{r}_{B}\right)$ is the potential:

$$
V\left(\mathbf{r}_{A}-\mathbf{r}_{B}\right)=-v_{0} \delta\left(\mathbf{r}_{A}-\mathbf{r}_{B}\right) \quad v_{0}>0
$$

The sign of $v_{0}$ has been chosen in such a way that the interaction associated to the potential $V(\mathbf{r})$ is repulsive. The parameters $c$ and $L$ determine the average length of the trajectories of the polymers. The ends of the polymers are supposed to be fixed on two surfaces perpendicular to the $z$-axis and located at the heights $z=0$ and $z=L$. Both polymers have a preferred direction along the $z$ direction. The vectors $\mathbf{r}_{A}(z)$ and $\mathbf{r}_{B}(z), 0 \leq z \leq L$, measure the polymer displacement along the remaining two directions of the space. 
The action of Eq. (11) resembles that of two quantum particles in the case of imaginary time $z$. To stress these analogies with quantum mechanics, the $z$-variable will be treated as a pseudo-time and renamed using from now on the letter $t$ instead of $z$.

In the system of the center of mass:

$$
\mathbf{r}=\mathbf{r}_{A}-\mathbf{r}_{B} \quad \mathbf{R}=\frac{\mathbf{r}_{A}+\mathbf{r}_{B}}{2}
$$

the action (11) becomes:

$$
\mathcal{A}_{0}=\int_{0}^{L} d t\left[\frac{c}{2}\left(\frac{d \mathbf{r}}{d t}\right)^{2}+2 c\left(\frac{d \mathbf{R}}{d t}\right)^{2}-V(\mathbf{r})\right]
$$

The motion of the center of mass, which is a free motion described by the coordinate $\mathbf{R}(t)$, will be ignored.

We consider the partition function of the above two-polymer system with the addition of a constraint on the entanglement of the trajectories:

$$
\mathcal{Z}_{m}=\int \mathcal{D} \mathbf{r} e^{-\int_{0}^{L} d t\left[\frac{c}{2}\left(\frac{d r}{d t}\right)+V(r)\right]} \delta(m-\chi)
$$

$\chi$ is the so-called winding angle. Its expression is given by:

$$
\chi=\int_{0}^{L} \mathbf{A}(\mathbf{r}(t)) \cdot d \mathbf{r}(t)
$$

where $\mathbf{A}(\mathbf{r})$ is a vector potential with components:

$$
A_{j}(\mathbf{r})=\frac{1}{2 \pi} \epsilon_{i j} \frac{x^{i}}{\mathbf{r}^{2}} \quad i, j=1,2
$$

In the above equation we have represented the vector $\mathbf{r}$ using cartesian coordinates $x^{1}, x^{2}$. i. e. $\mathbf{r}=\left(x^{1}, x^{2}\right)$. Moreover, from now on, middle latin indices $i, j, \ldots=1,2$ will label the directions which are perpendicular to the $t$-axis. The definition of the partition function $\mathcal{Z}_{m}$ is completed by the boundary conditions at $t=0$ and $t=L$ :

$$
\mathbf{r}(0)=\mathbf{r}_{0} \quad \mathbf{r}(L)=\mathbf{r}_{1}
$$

The quantity in Eq. (6) becomes a topological invariant if the polymer trajectories are closed. In the present case, in which the trajectories are open, $\chi$ just counts the angle with which one polymer winds up around the other. Thus, the partition function $\mathcal{Z}_{m}$ gives the formation probability of polymer paths winding up of an angle

$$
\Delta \theta=2 \pi m
$$


Exploiting the Fourier representation of Dirac $\delta$-functions

$$
\delta(m-\chi)=\int_{-\infty}^{+\infty} \frac{d \lambda}{2 \pi} e^{i \lambda(m \chi)}
$$

Eq. (5) can be rewritten as follows:

$$
\mathcal{Z}_{m}=\int_{-\infty}^{+\infty} \frac{d \lambda}{2 \pi} e^{i m \lambda} \mathcal{Z}_{\lambda}
$$

where

$$
\mathcal{Z}_{\lambda}=\int \mathcal{D} \mathbf{r} e^{-\int_{0}^{L} d t \mathcal{L}}
$$

The Lagrangian $\mathcal{L}$ is that of a particle immersed in the magnetic potential associated to the vector field (7):

$$
\mathcal{L}=\frac{c}{2}\left(\frac{d \mathbf{r}}{d t}\right)^{2}+i \lambda \frac{d \mathbf{r}}{d t} \cdot \mathbf{A}-V(\mathbf{r})
$$

The Fourier transformed partition function $\mathcal{Z}_{\lambda}$ is the grand canonical version of the original partition function $\mathcal{Z}_{m}$, in which the number $m$ is allowed to take any possible value.

$\mathcal{Z}_{\lambda}$ coincides with the propagator $\mathcal{G}_{\lambda}\left(L ; \mathbf{r}_{1}, \mathbf{r}_{0}\right)$, which satisfies the following pseudoSchrödinger equation:

$$
\left[\frac{\partial}{\partial L}-\mathcal{H}\right] \mathcal{G}_{\lambda}\left(L ; \mathbf{r}_{1}, \mathbf{r}_{0}\right)=0
$$

$\mathcal{H}$ is the Hamiltonian of the system, computed starting from the Lagrangian (13):

$$
\mathcal{H}=\frac{1}{2 c}(\boldsymbol{\nabla}-i \lambda \mathbf{A})^{2}+V(\mathbf{r})
$$

Eq. (14) is completed by the boundary condition at $L=0$ :

$$
\mathcal{G}_{\lambda}\left(0 ; \mathbf{r}_{1}, \mathbf{r}_{0}\right)=\delta\left(\mathbf{r}_{1}-\mathbf{r}_{0}\right)
$$

The average degree of entanglement of the two polymers can be estimated computing the topological moments of the winding angle $\left\langle m^{2 k}\right\rangle \mathbf{r}_{1}, \mathbf{r}_{0}, k=0,1,2, \ldots[22]$. Once the partition function is known, the $\left\langle m^{2 k}\right\rangle \mathbf{r}_{1}, \mathbf{r}_{0}$ may be expressed as follows:

$$
\left\langle m^{2 k}\right\rangle \mathbf{r}_{1}, \mathbf{r}_{0}=\frac{\int_{-\infty}^{+\infty} d m m^{2 k} \mathcal{Z}_{m}}{\int_{-\infty}^{+\infty} d m \mathcal{Z}_{m}}=\frac{\int_{-\infty}^{+\infty} d m m^{2 k} \int_{-\infty}^{+\infty} \frac{d \lambda}{2 \pi} e^{i m \lambda} \mathcal{G}_{\lambda}\left(L ; \mathbf{r}_{1}, \mathbf{r}_{0}\right)}{\int_{-\infty}^{+\infty} d m \int_{-\infty}^{+\infty} \frac{d \lambda}{2 \pi} e^{i m \lambda} \mathcal{G}_{\lambda}\left(L ; \mathbf{r}_{1}, \mathbf{r}_{0}\right)}
$$

The quantities $\left\langle m^{2 k}\right\rangle \mathbf{r}_{1}, \mathbf{r}_{0}$ depend on the boundary conditions $\mathbf{r}_{0}, \mathbf{r}_{1}$ and, of course, on the parameters $c$ and $L$. For practical reasons, we will also consider the following averaged topological moments:

$$
\left\langle m^{2 k}\right\rangle=\frac{\int d^{2} r_{0} \int d^{2} r_{1} \int d m m^{2 k} \mathcal{Z}_{m}}{\int d^{2} r_{0} \int d^{2} r_{1} \int d m \mathcal{Z}_{m}}
$$


As Eq. (18) shows, the average is performed with respect to the relative positions $\mathbf{r}_{0}, \mathbf{r}_{1}$ of the endpoints. This is equivalent to an average over the positions of the endpoints $\mathbf{r}_{A}(t), \mathbf{r}_{B}(t)$ at the instants $t=0$ and $t=L$, because the coordinates of the center of mass have been factored out from the partition function and thus they do not play any role. The advantage of the averaged topological moments is that, a posteriori, it will be seen that their computation is easier than that of the topological moments given in Eq. (17). From the physical point of view, the averaged topological moments measure the entanglement of two polymers, whose ends at the instants $t=0$ and $t=L$ are free to move.

Here we will be interested only in the second moment $\left\langle m^{2}\right\rangle \mathbf{r}_{1}, \mathbf{r}_{0}$ and in the averaged second moment $\left\langle m^{2}\right\rangle$, i. e. in the case $k=1$ of Eqs. (17) and (18). The second moment is in fact enough in order to estimate the formation probability of entanglement with a given winding angle and to determine how the winding angle grows with increasing polymer lengths.

In the following it will be useful to work in the so-called energy representation, i. .e considering the Laplace transformed of the partition function $\mathcal{G}_{\lambda}\left(L ; \mathbf{r}_{1}, \mathbf{r}_{0}\right)$ with respect to $L:$

$$
\mathcal{G}_{\lambda}\left(E ; \mathbf{r}_{1}, \mathbf{r}_{0}\right)=\int_{0}^{+\infty} d L e^{-E L} \mathcal{G}_{\lambda}\left(L ; \mathbf{r}_{1}, \mathbf{r}_{0}\right)
$$

The new partition function $\mathcal{G}_{\lambda}\left(E ; \mathbf{r}_{1}, \mathbf{r}_{0}\right)$ describes the probability of two entangled polymers of any length subjected to the condition that the relative positions of the polymer end at the initial and final instants $t_{0}$ and $t_{1}$ are given by the vectors $\mathbf{r}_{0}$ and $\mathbf{r}_{1}$. With respect to the formulation in the $L$ - space, however, the distance $t_{1}-t_{0}$ is no longer exactly equal to $L$, but is allowed to vary according to a distribution which is governed by the Boltzmann-like factor $e^{E L}$. Thus, $E$ plays the role of the chemical potential conjugated to the end-to-end distance of the polymer trajectories in the $t$-direction. It is worth to remember that, roughly speaking, small values of $E$ correspond to large values of $L$, while large values of $E$ correspond to small values of $L$. Starting from Eq. (14) and recalling the boundary conditions (16), it is easy to check that $\mathcal{G}_{\lambda}\left(E ; \mathbf{r}_{1}, \mathbf{r}_{0}\right)$ satisfies the stationary pseudo-Schrödinger equation:

$$
[E-\mathcal{H}] \mathcal{G}_{\lambda}\left(E ; \mathbf{r}_{1}, \mathbf{r}_{0}\right)=\delta\left(\mathbf{r}_{1}-\mathbf{r}_{0}\right)
$$

where $\mathcal{H}$ is always the Hamiltonian of Eq. (15). 


\section{CALCULATION OF THE SECOND MOMENT USING THE FIELD THEORETICAL FORMULATION}

In this Section we wish to evaluate the expression of the second moment as a function of the energy $E$ using a field theoretical formulation of the polymer partition function. The starting point is provided by the formula of the second moment in the $L$-space suitably rewritten in the following way:

$$
\left\langle m^{2}\right\rangle \mathbf{r}_{1}, \mathbf{r}_{0}=\frac{N\left(L ; \mathbf{r}_{1}, \mathbf{r}_{0}\right)}{D\left(L ; \mathbf{r}_{1}, \mathbf{r}_{0}\right)}
$$

For consistency with Eq. (17), the numerator $N\left(L ; \mathbf{r}_{1}, \mathbf{r}_{0}\right)$ and the denominator $D\left(L ; \mathbf{r}_{1}, \mathbf{r}_{0}\right)$ appearing in Eq. (21) must be of the form:

$$
N\left(L ; \mathbf{r}_{1}, \mathbf{r}_{0}\right)=\int_{-\infty}^{+\infty} d m m^{2} \int_{-\infty}^{+\infty} \frac{d \lambda}{2 \pi} e^{i m \lambda} \mathcal{G}_{\lambda}\left(L ; \mathbf{r}_{1}, \mathbf{r}_{0}\right)
$$

and

$$
D\left(L ; \mathbf{r}_{1}, \mathbf{r}_{0}\right)=\int_{-\infty}^{+\infty} d m \int_{-\infty}^{+\infty} \frac{d \lambda}{2 \pi} e^{i m \lambda} \mathcal{G}_{\lambda}\left(L ; \mathbf{r}_{1}, \mathbf{r}_{0}\right)
$$

Using Eq. (19), it is now straightforward to compute the Laplace transform of $N\left(L ; \mathbf{r}_{1}, \mathbf{r}_{0}\right)$ and $D\left(L ; \mathbf{r}_{1}, \mathbf{r}_{0}\right)$ :

$$
\begin{gathered}
N\left(E ; \mathbf{r}_{1}, \mathbf{r}_{0}\right)=\int_{-\infty}^{+\infty} d m m^{2} \int_{\infty}^{+\infty} \frac{d \lambda}{2 \pi} e^{i m \lambda} \mathcal{G}_{\lambda}\left(E ; \mathbf{r}_{1}, \mathbf{r}_{0}\right) \\
D\left(E ; \mathbf{r}_{1}, \mathbf{r}_{0}\right)=\int_{-\infty}^{+\infty} d m \int_{\infty}^{+\infty} \frac{d \lambda}{2 \pi} e^{i m \lambda} \mathcal{G}_{\lambda}\left(E ; \mathbf{r}_{1}, \mathbf{r}_{0}\right)
\end{gathered}
$$

Once the functions $N\left(E ; \mathbf{r}_{1}, \mathbf{r}_{0}\right)$ and $D\left(E ; \mathbf{r}_{1}, \mathbf{r}_{0}\right)$ are known, one can construct the ratio:

$$
\left\langle m^{2}\right\rangle \mathbf{r}_{1}, \mathbf{r}_{0}(E)=\frac{N\left(E ; \mathbf{r}_{1}, \mathbf{r}_{0}\right)}{D\left(E ; \mathbf{r}_{1}, \mathbf{r}_{0}\right)}
$$

which is nothing but the second moment of the winding angle expressed as a function of the chemical potential $E$.

We remark that the Green function $\mathcal{G}_{\lambda}\left(E ; \mathbf{r}_{1}, \mathbf{r}_{0}\right)$ is related to the Feynman propagator of the spin- $\frac{1}{2}$ Aharonov-Bohm problem in quantum mechanics. In principle, this Green function can be computed exactly starting from Eq. (201) [29], but its final expression is too complicated for our purposes. Moreover, the method used in [29] to renormalize the singularities coming from the presence of the $\delta$-function potential is valid only in a restricted region of the domain of $\lambda$. This is incompatible with our requirements, because, to derive 
the second moment, one has to integrate $\mathcal{G}_{\lambda}\left(E ; \mathbf{r}_{1}, \mathbf{r}_{0}\right)$ with respect to $\lambda$ over the whole real line. For this reason, we prefer here to use a field theoretical representation of this Green function. This is achieved by noting that $\mathcal{G}_{\lambda}\left(E ; \mathbf{r}_{1}, \mathbf{r}_{0}\right)$ coincides with the inverse matrix element of the operator $E-\mathcal{H}$ :

$$
\mathcal{G}_{\lambda}\left(E ; \mathbf{r}_{1}, \mathbf{r}_{0}\right)=\left\langle\mathbf{r}_{1}\left|\frac{1}{E-\mathcal{H}}\right| \mathbf{r}_{0}\right\rangle
$$

and may be expressed in a functional integral form in terms of replica fields:

$$
\mathcal{G}_{\lambda}\left(E ; \mathbf{r}_{1}, \mathbf{r}_{0}\right)=\lim _{n \rightarrow 0} \int \mathcal{D} \Psi \mathcal{D} \Psi^{*} \psi_{1}\left(\mathbf{r}_{1}\right) \psi_{1}^{*}\left(\mathbf{r}_{0}\right) e^{-S\left(\Psi^{*}, \Psi\right)}
$$

In the above equation $\Psi^{*}, \Psi$ are multiplets of replica fields:

$$
\Psi=\left(\psi_{1}, \ldots, \psi_{n}\right) \quad \Psi^{*}=\left(\psi_{1}^{*}, \ldots, \psi_{n}^{*}\right)
$$

with action

$$
S\left(\Psi^{*}, \Psi\right)=\int d^{2} x \Psi^{*} \star\left[E-\frac{1}{2 c}\left(\nabla_{x}-i \lambda \mathbf{A}\right)^{2}-v_{0} \delta(x)\right] \Psi
$$

The symbol $\star$ in Eq. (28) denotes summation over the replica index. For example $\Psi^{*} \star \Psi=$ $\sum_{\sigma=1}^{n} \psi_{\sigma}^{*} \psi_{\sigma}$. Below it will be used also the convention $\Psi^{*} \star \Psi=|\Psi|^{2}$. The details of the derivation of Eq. (28) can be found in previous publications on the subject [12, 29] and will not be provided here.

In order to proceed, it will be convenient to expand the action (30) in powers of $\lambda$ :

$$
S\left(\Psi^{*}, \Psi\right)=S_{0}\left(\Psi^{*}, \Psi\right)+\lambda S_{1}\left(\Psi^{*}, \Psi\right)+\lambda^{2} S_{2}\left(\Psi^{*}, \Psi\right)
$$

where we have put:

$$
\begin{gathered}
S_{0}\left(\Psi^{*}, \Psi\right)=\int d^{2} x\left[\frac{1}{2 c}|\nabla \Psi|^{2}+\left(E-v_{0} \delta(x)\right)|\Psi|^{2}\right] \\
S_{1}\left(\Psi^{*}, \Psi\right)=\frac{i}{2 c} \int d^{2} x \mathbf{A} \cdot\left[\Psi^{*} \star(\nabla \Psi)-\left(\nabla \Psi^{*}\right) \star \Psi\right] \\
S_{2}\left(\Psi^{*}, \Psi\right)=\frac{1}{2 c} \int d^{2} x \mathbf{A}^{2}|\Psi|^{2}
\end{gathered}
$$

At this point we come back to the computation of the quantities $N\left(E ; \mathbf{r}_{1}, \mathbf{r}_{0}\right)$ and $D\left(E ; \mathbf{r}_{1}, \mathbf{r}_{0}\right)$ appearing in the expression of the second moment. Exploiting the new form of the partition function given by Eqs. (28-34), together with the relation

$$
\int_{-\infty}^{+\infty} d m m^{\nu} e^{i m \lambda}=2 \pi(i)^{\nu} \frac{\partial^{\nu} \delta(\lambda)}{\partial \lambda^{\nu}} \quad \nu=0,1, \ldots
$$


and the fact that $\mathcal{Z}_{ \pm \infty}=0$, it is possible to rewrite Eqs. (24) and (25) as follows [44]:

$$
\begin{gathered}
N\left(E ; \mathbf{r}_{1}, \mathbf{r}_{0}\right)=\lim _{n \rightarrow 0} \int \mathcal{D} \Psi^{*} \mathcal{D} \Psi \psi_{1}\left(\mathbf{r}_{1}\right) \psi_{1}^{*}\left(\mathbf{r}_{0}\right)\left[2 S_{2}\left(\Psi^{*}, \Psi\right)-\left(S_{1}\left(\Psi^{*}, \Psi\right)\right)^{2}\right] e^{-S_{0}\left(\Psi^{*}, \Psi\right)} \\
D\left(E, \mathbf{r}_{1}, \mathbf{r}_{0}\right)=\lim _{n \rightarrow 0} \int \mathcal{D} \Psi^{*} \mathcal{D} \Psi \psi_{1}\left(\mathbf{r}_{1}\right) \psi_{1}^{*}\left(\mathbf{r}_{0}\right) e^{-S_{0}\left(\Psi^{*}, \Psi\right)}
\end{gathered}
$$

The right hand sides of Eqs. (36) and (37) represent vacuum expectation values of a field theory governed by the action $S_{0}\left(\Psi^{*}, \Psi\right)$ of Eq. (32). In the formulation in terms of quantum operators we have:

$$
\begin{gathered}
N\left(E ; \mathbf{r}_{1}, \mathbf{r}_{0}\right)=\lim _{n \rightarrow 0}\left\langle 0\left|\psi_{1}\left(\mathbf{r}_{1}\right) \psi_{1}^{*}\left(\mathbf{r}_{0}\right) 2 S_{2}\left(\Psi^{*}, \Psi\right)\right| 0\right\rangle_{n}-\lim _{n \rightarrow 0}\left\langle 0\left|\psi_{1}\left(\mathbf{r}_{1}\right) \psi_{1}^{*}\left(\mathbf{r}_{0}\right)\left(S_{1}\left(\Psi^{*}, \Psi\right)\right)^{2}\right| 0\right\rangle_{n} \\
D\left(E ; \mathbf{r}_{1}, \mathbf{r}_{0}\right)=\lim _{n \rightarrow 0}\left\langle 0\left|\psi_{1}\left(\mathbf{r}_{1}\right) \psi_{1}^{*}\left(\mathbf{r}_{0}\right)\right| 0\right\rangle_{n}
\end{gathered}
$$

The correlation functions have a subscript $n$ to remember that, according to the replica method, they should be computed first assuming that the number of replicas $n$ is an arbitrary positive integer and then taking the limit for $n$ going to zero.

The above correlators may be evaluated using standard field theoretical methods. One could be tempted to use a perturbative approach assuming that the value of $v_{0}$ appearing in the action $S_{0}\left(\Psi^{*}, \Psi\right)$ of Eqs. (36) and (37) is small, but this is not necessary. As a matter of fact, if it is true that $S_{0}\left(\Psi^{*}, \Psi\right)$ does not describe free fields because of the presence of the $\delta$-function potential, it is also true that it is just quadratic in the fields. As a consequence, one is allowed to define a propagator $G(E ; \mathbf{x}, \mathbf{y})$ associated with this action. It is easy to check that $G(E ; \mathbf{x}, \mathbf{y})$ satisfies the equation:

$$
\left[E-\frac{1}{2 c} \nabla_{\mathbf{x}}^{2}-v_{0} \delta(\mathbf{x})\right] G(E ; \mathbf{x}, \mathbf{y})=\delta(\mathbf{x}, \mathbf{y})
$$

Using the above propagator, one can evaluate the amplitudes in Eqs. (38) and (39) exactly by contracting the fields in all possible ways according to the Wick theorem. After straightforward calculations, one finds:

$$
\begin{gathered}
\lim _{n \rightarrow 0}\left\langle 0\left|\psi_{1}\left(\mathbf{r}_{1}\right) \psi_{1}^{*}\left(\mathbf{r}_{0}\right)\right| 0\right\rangle_{n}=G\left(E ; \mathbf{r}_{1}, \mathbf{r}_{0}\right) \\
\lim _{n \rightarrow 0}\left\langle 0\left|\psi_{1}\left(\mathbf{r}_{1}\right) \psi_{1}^{*}\left(\mathbf{r}_{0}\right) S_{2}\left(\Psi^{*}, \Psi\right)\right| 0\right\rangle_{n}=K\left(\mathbf{r}_{1}, \mathbf{r}_{0}\right) \\
\lim _{n \rightarrow 0}\left\langle 0\left|\psi_{1}\left(\mathbf{r}_{1}\right) \psi^{*}\left(\mathbf{r}_{0}\right)\left(S_{1}\left(\Psi^{*}, \Psi\right)\right)^{2}\right| 0\right\rangle_{n}=I_{1}\left(\mathbf{r}_{1}, \mathbf{r}_{0}\right)+I_{2}\left(\mathbf{r}_{1}, \mathbf{r}_{0}\right)+I_{3}\left(\mathbf{r}_{1}, \mathbf{r}_{0}\right)+I_{4}\left(\mathbf{r}_{1}, \mathbf{r}_{0}\right)
\end{gathered}
$$


where

$$
\begin{aligned}
& K\left(\mathbf{r}_{1}, \mathbf{r}_{0}\right)=\frac{1}{2 c} \int d^{2} x \mathbf{A}^{2}(\mathbf{x}) G\left(E ; \mathbf{r}_{1}, \mathbf{x}\right) G\left(E ; \mathbf{x}, \mathbf{r}_{0}\right) \\
& I_{1}\left(\mathbf{r}_{1}, \mathbf{r}_{0}\right)=-\frac{1}{2 c^{2}} \int d^{2} x d^{2} y\left[A_{i}(\mathbf{x}) G\left(E ; \mathbf{x}, \mathbf{r}_{1}\right)\left(\nabla_{\mathbf{x}}^{i} G(E ; \mathbf{y}, \mathbf{x})\right) A_{j}(\mathbf{y})\left(\nabla_{\mathbf{y}}^{j} G\left(E ; \mathbf{r}_{0}, \mathbf{y}\right)\right)\right] \\
& I_{2}\left(\mathbf{r}_{1}, \mathbf{r}_{0}\right)=+\frac{1}{2 c^{2}} \int d^{2} x d^{2} y\left[A_{i}(\mathbf{x})\left(\nabla_{\mathbf{x}}^{i} G\left(E ; \mathbf{x}, \mathbf{r}_{1}\right)\right) G(E ; \mathbf{y}, \mathbf{x}) A_{j}(\mathbf{y})\left(\nabla_{\mathbf{y}}^{j} G\left(E ; \mathbf{r}_{0}, \mathbf{y}\right)\right)\right] \\
& I_{3}\left(\mathbf{r}_{1}, \mathbf{r}_{0}\right)=+\frac{1}{2 c^{2}} \int d^{2} x d^{2} y\left[A_{i}(\mathbf{x}) G\left(E ; \mathbf{r}_{0}, \mathbf{x}\right)\left(\nabla_{\mathbf{x}}^{i} \nabla_{\mathbf{y}}^{j} G(E ; \mathbf{x}, \mathbf{y})\right) A_{j}(\mathbf{y}) G\left(E ; \mathbf{y}, \mathbf{r}_{1}\right)\right] \\
& I_{4}\left(\mathbf{r}_{1}, \mathbf{r}_{0}\right)=-\frac{1}{2 c^{2}} \int d^{2} x d^{2} y\left[A_{i}(\mathbf{x}) G\left(E ; \mathbf{r}_{0}, \mathbf{x}\right)\left(\nabla_{\mathbf{x}}^{i} G(E ; \mathbf{x}, \mathbf{y})\right) A_{j}(\mathbf{y})\left(\nabla_{\mathbf{y}}^{j} G\left(E ; \mathbf{y}, \mathbf{r}_{1}\right)\right)\right]
\end{aligned}
$$

From the physical point of view, the above equations may be interpreted in the following way. The fields $\Psi(\mathbf{x})$ and $\Psi^{*}(\mathbf{x})$ contain operators which, inside each replica sector, create and annihilate segments of the two polymers, whose relative positions are given by the vector $\mathbf{x}$. The two polymer system has been projected in the two-dimensional plane perpendicular to the $t$-axis. For this reason, there appear only the transverse coordinates $\mathbf{x}$. The only remnant of the third dimension is the dependence on the energy $E$. The correlation functions (42) and (43) describe the fluctuations of the two polymers immersed in the $\delta$-function potential and subjected to the interactions represented by the vector potential (7). We recall that the origin of the latter interactions is the presence of the constraint on the winding angle in the partition function (5). To evaluate the correlation functions (42) and (43), one needs to consider only a finite number of Feynman diagrams, corresponding to the relevant processes with which the two polymers interact together. The result, after the analytical evaluation of these diagrams, is provided by Eqs. (45) 48). Let us note that in these equations the repulsive interactions due to the $\delta$-function potential are hidden in the propagators $G(E ; \mathbf{x}, \mathbf{y})$. The Feynman diagrams related to the amplitudes of Eqs. (45)(48) are all topologically equivalent to the diagram of Fig. 11. The amplitude of Eq. (44) is related instead to the Feynman diagram of Fig. 2. The vectors $\mathbf{r}_{1}$ and $\mathbf{r}_{0}$ denote the relative positions of the end points of the two polymers at the initial and final instants, as already mentioned. The integration variables $\mathbf{x}$ and $\mathbf{y}$ appearing in Eqs. (4448) may be regarded as the vectors which give the relative positions of the trajectories of the two polymers at the instants in which they interact together via the external vector potential A of Eq. (17). There is no restriction on the domain of integration of $\mathbf{x}$ and $\mathbf{y}$, so that the components of these relative vectors are allowed to take any value. This implies that the distance between the polymer segments when the interaction with $\mathbf{A}$ occurs can be arbitrarily large. 


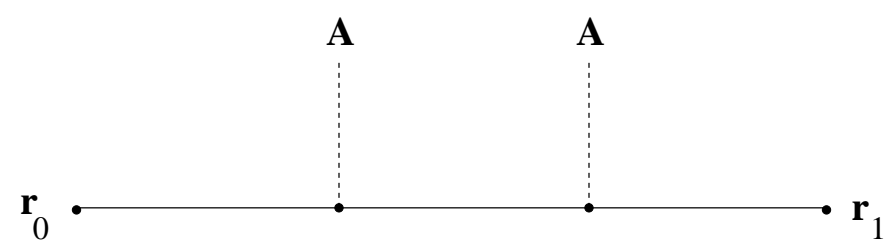

FIG. 1: Feynman diagram corresponding to the amplitudes of Eqs. (45)-(48). The two polymers $A$ and $B$ start at a distance $\left|\mathbf{r}_{0}\right|$ from each other and interact twice with the the external field A. At the end, the relative position of the end points at the instant $t=L$ is given by $\mathbf{r}_{1}$. The three-vertices appearing in the Figure are related to the interaction described by Eq. (33).

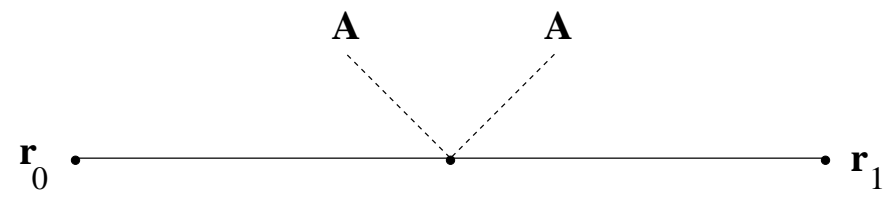

FIG. 2: Feynman diagram corresponding to the amplitude of Eq. (44). The two polymers $A$ and $B$ start at a distance $\left|\mathbf{r}_{0}\right|$ from each other and interact with the the external field $\mathbf{A}$. At the final instant $t=L$, the relative position of the end points is given by $\mathbf{r}_{1}$. The four-vertex appearing in this Figure is related to the interaction described by Eq. (34).

Now that the correlation functions which are present in the expressions of $N\left(E ; \mathbf{r}_{1}, \mathbf{r}_{0}\right)$ and $D\left(E ; \mathbf{r}_{1}, \mathbf{r}_{0}\right)$ given in Eqs. (38) and (39) have been evaluated, see Eqs. (44) 48), we may put everything together and give to the second moment of Eq. (26) a more explicit form:

$$
\left\langle m^{2}\right\rangle_{\mathbf{r}_{1}, \mathbf{r}_{0}}=\frac{2 K\left(\mathbf{r}_{0}, \mathbf{r}_{0}\right)-\sum_{\omega=1}^{4} I_{\omega}\left(\mathbf{r}_{0}, \mathbf{r}_{0}\right)}{G\left(E ; \mathbf{r}_{0}, \mathbf{r}_{0}\right)}
$$

In conclusion, the initial problem of computing the second moment of the winding angle $\left\langle m^{2}\right\rangle \mathbf{r}_{1}, \mathbf{r}_{0}$ has been reduced to the evaluation of a finite number of integrals, which are given in Eqs. (44-48). Of course, to make these integrals really explicit, we still need to derive the propagator $G(E ; \mathbf{x}, \mathbf{y})$, which is so far the only missing ingredient. This will be done in the next Section. 


\section{GREEN FUNCTIONS IN THE CASE OF HAMILTONIANS WITH A $\delta$-FUNCTION POTENTIAL}

Let $G_{0}(L ; \mathbf{x}, \mathbf{y})$ be the solution of the differential equation:

$$
\left(\frac{\partial}{\partial L}-\mathcal{H}_{0}\right) G_{0}(L ; \mathbf{x}, \mathbf{y})=0
$$

for a given Hamiltonian $\mathcal{H}_{0}$. When $L=0, G_{0}(L ; \mathbf{x}, \mathbf{y})$ satisfies the boundary condition:

$$
G_{0}(0 ; \mathbf{x}, \mathbf{y})=\delta(\mathbf{x}-\mathbf{y})
$$

In the case of a Hamiltonian $\mathcal{H}$, obtained by adding to $\mathcal{H}_{0}$ a $\delta$-function potential as a perturbation:

$$
\mathcal{H}(\mathbf{x})=\mathcal{H}_{0}(\mathbf{x})-v_{0} \delta(\mathbf{x})
$$

we consider the analogous differential problem:

$$
\begin{gathered}
\left(\frac{\partial}{\partial L}-\mathcal{H}\right) G(L ; \mathbf{x}, \mathbf{y})=0 \\
G(0 ; \mathbf{x}, \mathbf{y})=\delta(\mathbf{x}-\mathbf{y})
\end{gathered}
$$

We wish to compute $G(L ; \mathbf{x}, \mathbf{y})$ starting from the Green function $G_{0}(L ; \mathbf{x}, \mathbf{y})$, which is supposed to be known. It is possible to show that $G(L ; \mathbf{x}, \mathbf{y})$ and $G_{0}(L ; \mathbf{x}, \mathbf{y})$ are related by the integral equation [30, 31]:

$$
G(L ; \mathbf{x}, \mathbf{y})=G_{0}(L ; \mathbf{x}, \mathbf{y})-v_{0} \int_{0}^{L} d s \int d^{2} z G_{0}(L-s ; \mathbf{x}, \mathbf{z}) \delta(\mathbf{z}) G(s ; \mathbf{z}, \mathbf{y})
$$

We see that in the right hand side of the above equation the presence of the $\delta$-function forces us to consider the functions $G_{0}(L ; \mathbf{x}, \mathbf{y})$ and $G(L ; \mathbf{x}, \mathbf{y})$ evaluated at the points $\mathbf{x}=0$ and $/$ or $\mathbf{y}=0$. Usually, at these points Green functions may be not well defined due to the presence of singularities. A concrete procedure to remove these singularities will be indicated later. For the moment, we go further with formal manipulations, assuming that some kind of consistent regularization of the possible divergences has been introduced.

First of all, we perform the integration over $d^{2} z$ in Eq. (55):

$$
G(L ; \mathbf{x}, \mathbf{y})=G_{0}(L ; \mathbf{x}, \mathbf{y})-v_{0} \int_{0}^{L} d s G_{0}(L-s ; \mathbf{x}, 0) G(s ; 0, \mathbf{y})
$$


The integral in $d s$ appearing in the right hand side of Eq. (56) is a convolution which can be better treated after a Laplace transform. Thus, we transform both sides of this equation with respect to $L$ :

$$
G(E ; \mathbf{x}, \mathbf{y})=G_{0}(E ; \mathbf{x}, \mathbf{y})-v_{0} G_{0}(E ; \mathbf{x}, 0) G(E ; 0, \mathbf{y})
$$

where

$$
G(E ; \mathbf{x}, \mathbf{y})=\int_{0}^{+\infty} e^{-E L} G(L ; \mathbf{x}, \mathbf{y}) d L
$$

and

$$
G_{0}(E ; \mathbf{x}, \mathbf{y})=\int_{0}^{+\infty} e^{-E L} G_{0}(L ; \mathbf{x}, \mathbf{y}) d L
$$

At this point, it is easy to extract from Eq. (57) the expression of $G(E ; \mathbf{x}, \mathbf{y})$ :

$$
G(E ; \mathbf{x}, \mathbf{y})=G_{0}(E ; \mathbf{x}, \mathbf{y})-\frac{G_{0}(E ; \mathbf{x}, 0) G_{0}(E ; 0, \mathbf{y})}{\frac{1}{v_{0}}+G_{0}(E ; 0,0)}
$$

The above formula may be used in order to solve Eq. (40). In this case, $\mathcal{H}_{0}$ coincides with the free action:

$$
\mathcal{H}_{0}=\frac{1}{2 c} \nabla^{2}
$$

and the function $G_{0}(E ; \mathbf{x}, \mathbf{y})$ is given by:

$$
G_{0}(E ; \mathbf{x}, \mathbf{y})=\frac{c}{\pi} K_{0}(\sqrt{2 E c}|\mathbf{x}-\mathbf{y}|)
$$

Here $K_{0}(z)$ denotes the modified Bessel function of the second kind of order zero.

Clearly, we cannot apply directly Eq. (60) without introducing a regularization. As a matter of fact, if not treated, the naive denominator in the second term of the right hand side is equal to infinity, i. e. $\frac{1}{v_{0}}+G_{0}(E ; 0,0)=+\infty$. This is due to the fact that $K_{0}(z)$ diverges logarithmically in the limit $z \rightarrow 0$ :

$$
K_{0}(z) \sim-\log z \quad \text { for } z \sim 0
$$

A natural regularization is suggested by the fact that, in polymer physics, it has no sense to consider lengths which are smaller than the size of the molecules which compose the polymers. Thus, it seems reasonable to regulate ultraviolet divergences by introducing a cut-off $a$ at short distances. The length $a$ is comparable with the molecular size. According to this prescription, by inserting the Green function of Eq. (62) in Eq. (60), we obtain:

$$
G(E ; \mathbf{x}, \mathbf{y}) \equiv \frac{c}{\pi} K_{0}(\sqrt{2 E c}|\mathbf{x}-\mathbf{y}|)-\left(\frac{c}{\pi}\right)^{2} \frac{K_{0}(\sqrt{2 E c}|\mathbf{x}|) K_{0}(\sqrt{2 E c}|\mathbf{y}|)}{\frac{1}{v_{0}}+\frac{c}{\pi} K_{0}(\sqrt{2 E c} a)}
$$


The symbol $\equiv$ means that the quantity in the left hand side of an equation has been replaced in the right hand side with its regulated version. The above Green function is what we need in order to evaluate explicitly the amplitudes of Eqs. (41,43).

The infinities coming from the $\delta$-function potential should be treated with some care in order to avoid ambiguities. For this reason, we would like to compare the naive prescription used here to derive Eq. (64) with the more rigorous procedure of renormalization. It is known in fact that the renormalization of the infinities coming from $\delta$-function interactions produces physically sensible results [32]. The divergences will be regulated introducing a cut-off $\Lambda$ in the momentum space. As a consequence, it will be convenient to express the free Green function of Eq. (62) in momentum space. To this purpose, we use the following formula:

$$
K_{0}(m|\mathbf{x}-\mathbf{y}|)=\frac{1}{2 \pi} \int d^{2} p \frac{e^{i \mathbf{p} \cdot(\mathbf{x}-\mathbf{y})}}{\mathbf{p}^{2}+m^{2}}
$$

To evaluate the Green function at the singular point $\mathbf{x}=\mathbf{y}=0$ we need to compute the following divergent integral:

$$
I(m)=\frac{1}{2 \pi} \int \frac{d^{2} p}{\mathbf{p}^{2}+m^{2}}
$$

Using the above cut-off prescription to eliminate the ultraviolet singularities we get, in the assumption $\Lambda^{2} \gg m^{2}$ :

$$
I(m) \sim \log \frac{\Lambda}{m}
$$

Now, according to the spirit of renormalization, we subtract the infinities from the bare parameters of the theory. In our case, after choosing an arbitrary mass scale $\mu$, which gives the renormalization point, we renormalize the bare coupling constant $v_{0}$. Actually, it will be better to call it $v_{\text {bare }}$ instead of $v_{0}$ in order to distinguish it from the effective coupling constant $v_{0}$ appearing in Eq. (64). The subtraction of infinities is performed in such a way that the quantity:

$$
\frac{1}{v_{\text {bare }}}-G_{0}(E ; 0,0)=\frac{1}{v_{\text {ren }}}+\frac{c}{2 \pi} \log \left(\frac{\Lambda^{2}}{\mu^{2}}\right)-\frac{c}{2 \pi} \log \left(\frac{m^{2}}{\mu^{2}}\right)
$$

becomes finite. We choose a sort of minimal subtraction scheme, in which the renormalized coupling constant $v_{\text {ren }}$ is related to the bare coupling constant $v_{\text {bare }}$ as follows:

$$
\frac{1}{v_{\text {bare }}}+\frac{c}{2 \pi} \log \left(\frac{\Lambda^{2}}{\mu^{2}}\right)=\frac{1}{v_{\text {ren }}}
$$


Applying the last two above equations back to Eq. (60), we get as a result:

$$
G(E ; \mathbf{x}, \mathbf{y})=\frac{c}{\pi} K_{0}(\sqrt{2 E c}|\mathbf{x}-\mathbf{y}|)-\left(\frac{c}{\pi}\right)^{2} \frac{K_{0}(\sqrt{2 E c}|\mathbf{x}|) K_{0}(\sqrt{2 E c}|\mathbf{y}|)}{\frac{1}{v_{r e n}}-\frac{c}{2 \pi} \log \left(\frac{2 E c}{\mu^{2}}\right)}
$$

Eqs. (64) and (70) are reciprocally compatible. In fact, since $a$ is very small, because it is the smallest possible length scale in our polymer problem, one can use the following approximation (see Eq. (63) ) in the denominator of the second term of Eq. (64):

$$
\frac{1}{v_{0}}+\frac{c}{\pi} K_{0}(\sqrt{2 E c} a) \sim \frac{1}{v_{0}}-\frac{c}{2 \pi} \log (2 E c a)
$$

Comparing with the analogous denominator in Eq. (70), it is possible to relate $a$ with the inverse of the mass $\mu$ :

$$
\mu^{2}=\frac{1}{a^{2}}
$$

Moreover, the effective coupling constant $v_{0}$ of Eq. (64) may be identified with the renormalized coupling constant $v_{r e n}$, which gives the strength of the repulsive interaction (2) at distance scales of order $a$.

Before concluding this Section, we make a small digression about the translational invariance of the free Hamiltonian (61) and consequently of the free Green function (62). Clearly, this is not the same translational invariance that was already present in the original action (11) due to the translational invariance of the potential (2). This new invariance is rather related to the fact that the physics of the two polymer system in the absence of any interaction does not change if we modify the relative positions of the polymer ends at $t=0$ and $t=L$ in a symmetric way. An example of such transformations is the translation of both ends of polymer $A$ at the initial and final points by a constant vector a:

$$
\begin{aligned}
& \mathbf{r}_{A}(0)=\mathbf{r}_{A}(0)+\mathbf{a} \\
& \mathbf{r}_{A}(L)=\mathbf{r}_{A}(L)+\mathbf{a}
\end{aligned}
$$

As a result of the translations (73) $t=0$ and $t=L$ changes as follows:

$$
\begin{aligned}
& \mathbf{r}_{0}^{\prime}=\mathbf{r}_{0}+\mathbf{a} \\
& \mathbf{r}_{1}^{\prime}=\mathbf{r}_{1}+\mathbf{a}
\end{aligned}
$$

Clearly the propagator (62) is invariant under the above transformations. This kind of invariance can be explained as follows. As far as the two polymers $A$ and $B$ do not interact, 
each of them may be treated as an independent system. If we translate for instance both ends of polymer $A$ at $t=0$ and $t=L$ in the symmetrical way shown by Eqs. (73) and (74), the number of configurations of polymer $A$ and consequently the configurational entropy of the whole system do not change, because the transformation is equivalent to a translation of polymer $A$ in the space. Of course, this invariance disappears as soon as the two polymers start to interact or if they are entangled together. Indeed, if one adds to the free Hamiltonian (61) a $\delta$-function potential, the propagator stops to be translational invariant as shown by the Green function of Eq. (60), which does not depend on the difference $\mathbf{x}-\mathbf{y}$.

\section{REPULSIVE FORCES AND WINDING ANGLES: QUALITATIVE AND QUANTITATIVE CONSIDERATIONS}

In principle we have at this point all the ingredients which are necessary to compute the second moment of Eq. (26). In Eqs. (38) and (39), in fact, the quantities $N\left(E ; \mathbf{r}_{1}, \mathbf{r}_{0}\right)$ and $D\left(E ; \mathbf{r}_{1}, \mathbf{r}_{0}\right)$ are written as linear combinations of the amplitudes of Eqs. (41]43), which can be explicitly evaluated using the propagator $G(E, \mathbf{u}, \mathbf{v})$ given in Eq. (64) [45] and the formulas of Eqs. (44-48). The remaining task is to perform the integrations over the coordinates $\mathbf{x}$ and $\mathbf{y}$ in Eqs. (44,48). From the analytical point of view, the evaluation of these integrals poses severe technical problems, which can be solved only with the help of drastic approximations. However, the difficulties become milder if we average the second moment over the endpoints of the polymers as shown in Eq. (18). In the energy representation, which we are using, this means that we have to consider the following averaged version of the second moment in Eq. (26) :

$$
\left\langle m^{2}\right\rangle(E)=\frac{N(E)}{D(E)}
$$

where

$$
\begin{aligned}
N(E) & =\int d^{2} r_{0} \int d^{2} r_{1} N\left(E ; \mathbf{r}_{1}, \mathbf{r}_{0}\right) \\
D(E) & =\int d^{2} r_{0} \int d^{2} r_{1} D\left(E ; \mathbf{r}_{1}, \mathbf{r}_{0}\right)
\end{aligned}
$$

Accordingly, we need to integrate the quantities $K\left(\mathbf{r}_{1}, \mathbf{r}_{0}\right)$ and $I_{\omega}\left(\mathbf{r}_{1}, \mathbf{r}_{0}\right), \omega=1, \ldots, 4$ of Eqs. (44-48) with respect to $\mathbf{r}_{1}$ and $\mathbf{r}_{0}$. Putting:

$$
K(E)=\int d^{2} r_{0} \int d^{2} r_{1} K\left(\mathbf{r}_{1}, \mathbf{r}_{0}\right)
$$




$$
I_{\omega}(E)=\int d^{2} r_{0} \int d^{2} r_{1} I_{\omega}\left(\mathbf{r}_{1}, \mathbf{r}_{0}\right) \quad \omega=1, \ldots, 4
$$

we obtain from Eqs. (42) and (43) the following expressions of $N(E)$ and $D(E)$ :

$$
\begin{aligned}
& N(E)=2 K(E)-\sum_{\omega=1}^{4} I_{\omega}(E) \\
& D(E)=\int d^{2} r_{0} d^{2} r_{1} G\left(E ; \mathbf{r}_{1}, \mathbf{r}_{0}\right)
\end{aligned}
$$

It will also be convenient to split the propagator $G(E ; \mathbf{u}, \mathbf{v})$ of Eq. (64) into two contributions:

$$
G(E ; \mathbf{u}, \mathbf{v})=G_{0}(E ; \mathbf{u}, \mathbf{v})+G_{1}(E ; \mathbf{u}, \mathbf{v})
$$

where $G_{0}(E ; \mathbf{u}, \mathbf{v})$ is the free propagator of Eq. (62), which is invariant with respect to the transformations (75) and (76), while

$$
G_{1}(E ; \mathbf{u}, \mathbf{v})=\frac{c}{\pi} \lambda(E) K_{0}(\sqrt{2 E c}|\mathbf{u}|) K_{0}(\sqrt{2 E c}|\mathbf{v}|)
$$

In the above equation we have isolated in the expression of $G_{1}(E ; \mathbf{u}, \mathbf{v})$ the factor:

$$
\lambda(E)=-\frac{c}{\pi}\left(\frac{1}{v_{0}}+\frac{c}{\pi} K_{0}(\sqrt{2 E c} a)\right)^{-1}
$$

It is clear that the origin of the term $G_{1}(E ; \mathbf{u}, \mathbf{v})$ in the propagator is due to presence of the $\delta$-function interaction (2) in the polymer action (11). In fact, if $v_{0}=0$, this term vanishes identically. Thus, using the splitting of the propagator of Eq. (84), it is now possible to separate in Eqs. (44 48) the contributions to entanglement given by repulsive forces.

It seems natural to expand the quantities $D(E), K(E)$ and $I_{\omega}(E)$ defined in Eqs. (79), (80) and (81) with respect to $\lambda(E)$ as follows:

$$
\begin{aligned}
& D(E)=D^{(0)}(E)+D^{(1)}(E) \\
& K(E)=K^{(0)}(E)+K^{(1)}(E)+K^{(2)}(E) \\
& I_{\omega}(E)=I_{\omega}^{(0)}(E)+I_{\omega}^{(1)}(E)+I_{\omega}^{(2)}(E)+I_{\omega}^{(3)}(E)
\end{aligned}
$$

where the superscript $(n)$, with $n=0,1,2,3$, denotes the order in $\lambda(E)$. There are no higher order terms with $n \geq 4$, so the above expansions are exact.

It is easy to show how $K(E)$ and the $I_{\omega}(E)$ 's depend on the pseudo-energy $E$. After a rescaling of the integration variables $\mathbf{r}_{1}, \mathbf{r}_{0}, \mathbf{x}$ and $\mathbf{y}$ in Eqs. (78) and (179), one finds in fact that:

$$
K^{(n)}(E)=\lambda^{n}(E) E^{-2} K^{(n)} \quad n=0,1,2
$$




$$
I_{\omega}^{(n)}(E)=\lambda^{n}(E) E^{-2} I_{\omega}^{(n)} \quad n=0,1,2,3
$$

where the factors $K^{(n)}$ 's and the $I_{\omega}^{(n)}$ 's are functions of the parameters $a$ and $c$, but not of $E$ or $v_{0}$. In fact, the coupling constant $v_{0}$ appears only inside the powers of $\lambda(E)$. Let us note in Eqs. (90) and (91) the presence of the overall factor $E^{-2}$ in Eqs. (90) and (91). Looking at Eq. (82), it is clear that the whole function $N(E)$ is characterized by the leading scaling behavior $N(E) \sim E^{-2}$. In the $L$-space, after an inverse Laplace transform, this behavior corresponds to the following scaling law, which is typical of ideal polymers: $N(L) \sim L$. The powers of $\lambda(E)$, appearing in the expressions of $K^{(n)}(E)$ and $I_{\omega}^{(n)}(E)$, introduce corrections to this leading behavior that are at most logarithmic in $E$. As a matter of fact, if the condition $2 E c a^{2} \ll 1$ is satisfied, we have that:

$$
\lambda(E) \sim-\frac{c}{\pi}\left(v_{0}^{-1}-\frac{c}{\pi} \log (\sqrt{2 E c} a)\right)^{-1}
$$

Naively, the above seems the only logarithmic correction which is possible in the expressions of $N(E)$ and $D(E)$ when $E$ is small. However, that this is not true. In fact, in deriving Eqs. (901) and (91), we have not considered the divergences which arise in some of the integrations over the variables $\mathbf{x}, \mathbf{y}, \mathbf{r}_{0}$ and $\mathbf{r}_{1}$. After regulating these divergences with some prescription, as for instance the ultraviolet cut-off $a$ used in Eq. (64), we will see in SectionVI that the naive rescaling of variables exploited in order to obtain Eqs. (90) and (91) does no longer work and one should add extra logarithmic factors to these equations.

Eqs. (90) and (91) may be also useful to study the case of polymers in confined geometries. As a matter of fact, for large values of $E$, one recovers the limit of small values of $L$, in which the region between the initial and final height is very narrow. Looking at Eqs. (90) and (91), it is clear that the only interesting corrections when $E$ is large come from the powers of $\lambda(E)$. To evaluate these corrections, one should note that the modified Bessel function of the second kind $K_{0}(z)$ goes very fast to zero for large values of $z$. As a consequence, already in the domain of parameters in which $2 E \mathrm{Ca}^{2} \geq 10$, it is possible to make the very interesting approximation

$$
\lambda(E) \sim-\frac{c}{\pi} v_{0}
$$

Unfortunately, it turns out that the values of the energy for which the above equation is satisfied are not physical, as it will be shown below.

Other useful information on the influence of repulsive forces on the winding angle can be obtained studying the form of the function $G_{1}(E ; \mathbf{u}, \mathbf{v})$ of Eq. (85). We remember in 
fact that all the effects of the repulsive forces are concentrated in this component of the propagator. Supposing for example that the value of $|\mathbf{u}|$ is very large, i. e.:

$$
|\mathbf{u}| \gg \frac{1}{\sqrt{2 E c}}
$$

we have the following approximate expression of $G_{1}(E ; \mathbf{u}, \mathbf{v})$ :

$$
G_{1}(E ; \mathbf{u}, \mathbf{v})=\frac{c}{\sqrt{2 \pi}} \lambda(E)(2 E c)^{1 / 4} e^{-\sqrt{2 E c}|\mathbf{u}|} K_{0}(\sqrt{2 E c}|\mathbf{v}|)
$$

A relation analogous to Eq. (95) may be written also for the variable v. In practice, Eq. (95) means that the repulsive interactions do not play any particularly relevant role in polymer configurations in which the ends of the trajectories at some point are very distant. This is not a surprise. If the trajectories at some height $t$ are very far from each other, they will have little or no chance to interact together via the repulsive interactions of Eq. (2), which are of short range. Eq. (95) gives the concrete law with which the contributions of the repulsive forces are suppressed in configurations of this kind. In particular, if the distance between the trajectories is much greater than the characteristic length scale

$$
l_{\text {rep }}=1 / \sqrt{2 E c}
$$

the influence of the repulsive forces ceases to be relevant. Of course, even if at some points the trajectories are very distant, polymers will always have a chance to get near enough to be able to interact if they are sufficiently long. As a consequence, we expect that the characteristic length $l_{r e p}$ increases with the increasing of the lengths of the trajectories. It is easy to check that this is exactly the case. To show that, let us consider the dependence of $l_{\text {rep }}$ on the polymer length. One parameter which determines this length is the distance $L$ between the ends of the polymers along the $t$-axis. Indeed, a trajectory connecting the two ends of a polymer must be very long if these ends are located at very distant heights. In the energy representation, large values of $L$ correspond to small values of $E$. For example, in the limit $E=0$, which corresponds to infinite polymer lengths, we have that $l_{\text {rep }}=\infty$, confirming our intuitive expectations. Another confirmation comes from Eq. (104) below, where a rough estimation of the behavior of $l_{\text {rep }}$ with respect to the distance $L$ is given. The dependence on $L$ is not the whole story. As a matter of fact, during their random walk in the $t$-direction from $t=0$ to $t=L$, polymers are also allowed to wander in the remaining two directions. Loosely speaking, the variations in the length of the trajectories associated 
to the fluctuations in these transverse directions are taken into account by the parameter c. Smaller values of $c$ correspond to longer trajectories and vice-versa, see [36]. It is now easy to realize from Eq. (96) that the characteristic length $l_{r e p}$ increases when $c$ decreases as expected. Taking into account all the above considerations, it is possible to conclude that repulsive forces give relevant contributions to the second moment only in the case of configurations of the system in which the trajectories of the two polymers are not too far from each other. As a matter of fact, in the propagators appearing in the amplitudes of Eqs. (44-48) all configurations in which the distance between the trajectories at some height in the $t$-axis is bigger than a few characteristic lengths $l_{\text {rep }}$ are exponentially suppressed according to Eq. (95). One may also add that this suppression becomes milder in the case of long polymers, because we have seen that the characteristic lengths $l_{\text {rep }}$ grows with the length of the polymers with a law which has been given in Eq. (104).

In the rest of this Section we will analyze some interesting limiting cases, in which repulsive interactions become particularly weak or strong. To this purpose, it would be appealing to consider the quantity $c^{-1} \lambda(E)$, where $\lambda(E)$ has been given in Eq. (86), as an energy dependent effective or running coupling constant of the repulsive interactions. This could be suggested by the expansions of Eqs. (87 89 ) and by the fact that the quantity $c^{-1} \lambda(E)$ has the right dimension to be a coupling constant. Indeed, we will see that there are cases in which the strength of $\lambda(E)$ really determines the strength of the repulsive forces. However, this is not true in general, as it should be because $\lambda(E)$ is just a parameter which has been factored out from the expression of $G_{1}(E ; \mathbf{u}, \mathbf{v})$ and thus its meaning does not coincide with that of a running coupling constant. Keeping that in mind, we start to study the perturbative regime, in which $v_{0}$ is very small. In the part of the propagator in which there are the contributions of the repulsive forces, i. e. the function $G_{1}(E ; \mathbf{u}, \mathbf{v}), v_{0}$ is present only inside $\lambda(E)$. Expanding this quantity in powers of $v_{0}$, we obtain:

$$
\lambda(E) \sim \frac{c}{\pi}\left(-v_{0}+\frac{c}{\pi} v_{0}^{2} K_{0}(\sqrt{2 E c} a)+\ldots\right)
$$

We see that, at the leading order in $v_{0}, \lambda(E)$ is proportional to $v_{0}$ and thus, as it could have been expected, $G_{1}(E ; \mathbf{u}, \mathbf{v})$ may be treated as a small perturbation with respect to the free propagator $G_{0}(E, \mathbf{u}, \mathbf{v})$. Let's now go back to Eq. (93). In that equation it turns out that $\lambda(E)$ has the same behavior as in the perturbative regime, even if $\mathrm{Eq}$ (93) has been derived in the hypothesis that $2 E c a^{2} \geq 10$, but without supposing that $v_{0}$ is small. Before 
dwelling upon the physical meaning of this coincidence, let's see what is the significance of the condition $2 E c a^{2} \geq 10$. To this purpose, we make the following approximations:

$$
L \sim E^{-1} \quad \frac{1}{c} \sim a
$$

As mentioned before, it is quite reasonable to assume that the length $L$ is proportional to the inverse of the energy $E$, while the second approximation implies that polymers are very flexible. For example, in polyethylene the Kuhn length $1 / c$ is of the order of molecular sizes. Exploiting Eq. (98), it turns out that the condition $2 E c a^{2} \geq 10$ is equivalent to the condition $L \leq \frac{a}{5}$. This would mean that our system is squeezed in a volume whose height $L$ is smaller than the size of a monomer. Clearly, this situation is not very physical.

Since we have been able to compute the exact form of the propagator $G(E ; \mathbf{u}, \mathbf{v})$, it is not difficult to study also the strong coupling limit $v_{0} \longrightarrow \infty$. As in the perturbative case, the only affected part of the propagator (64) is the factor $\lambda(E)$ appearing in $G_{1}(E ; \mathbf{u}, \mathbf{v})$. After a trivial calculation, one finds that, in the strong coupling limit, the form of $G_{1}(E ; \mathbf{u}, \mathbf{v})$ is given by:

$$
G_{1}(E ; \mathbf{u}, \mathbf{v}) \sim\left(K_{0}(\sqrt{2 E c} a)\right)^{-1} K_{0}(\sqrt{2 E c}|\mathbf{u}|) K_{0}(\sqrt{2 E c}|\mathbf{v}|)
$$

Assuming that polymers are very long, let us study the left hand side of the above equation. This is a ratio of modified Bessel functions of the second kind. Since $a$ is a very small quantity and these functions have a logarithmic singularity if their argument is small, see Eq. (63), it is licit to suppose that

$$
K_{0}(\sqrt{2 E c} a)>K_{0}(\sqrt{2 E c}|\mathbf{u}|) K_{0}(\sqrt{2 E c}|\mathbf{v}|)
$$

unless $|\mathbf{u}| \sim a$ and/or $|\mathbf{v}| \sim a$. On the other side, we know from Eq. (94) that, if $|\mathbf{u}|,|\mathbf{v}| \gg \frac{1}{\sqrt{2 E c}}$, the product of modified Bessel functions $K_{0}(\sqrt{2 E c}|\mathbf{u}|) K_{0}(\sqrt{2 E c}|\mathbf{v}|)$ decays exponentially. In other words, in the left hand side of Eq. (99) the denominator will dominate over the numerator whenever the distance between the polymer trajectories is not of the order of a few molecular sizes. Thus, if $v_{0}$ is large, the major contributions to winding angle coming from the repulsive interactions occur when the trajectories are very near to each other. This could be expected from the fact that, in the strong coupling limit, one recovers the excluded volume interactions.

Finally, let us study the domain of the parameters $E$ and $c$ in which the condition

$$
2 E c a^{2} \ll 1
$$


is verified. We will see that this domain is particularly interesting, because if condition (101) is verified, the corrections of the repulsive interactions to the entropy dominated behavior of ideal polymers become relevant. It has been already shown that under the assumption made in Eq. (101), the parameter $\lambda(E)$ is approximated as in Eq. (92). Even if it is not strictly necessary, we suppose here that $v_{0}$ has some finite value, while polymers are so long that the following inequality is satisfied:

$$
v_{0}^{-1} \ll-\frac{c}{\pi} \log (\sqrt{2 E c} a)
$$

This further assumption is to eliminate the dependence on $v_{0}$, which could introduce confusion in the following discussion due to possible interferences of condition (101) with those of the perturbative and strong coupling regimes. In the $L$-space, Eq. (102) corresponds to the inequality $e^{2 \pi / c v_{0}} \ll \frac{L}{2 a}$. Now $G_{1}(E ; \mathbf{u}, \mathbf{v})$ may be approximated as follows:

$$
G_{1}(E ; \mathbf{u}, \mathbf{v}) \sim \frac{c}{\pi \log (\sqrt{2 E c} a)} K_{0}(\sqrt{2 E c}|\mathbf{u}|) K_{0}(\sqrt{2 E c}|\mathbf{v}|)
$$

As promised, the above equation does not contain the parameter $v_{0}$. We see from the left hand side of Eq. (103) that the function $G_{1}(E ; \mathbf{u}, \mathbf{v})$ is logarithmically suppressed, due to the presence of $\log (\sqrt{2 E c} a)$ in the denominator. This suppression effect is counterbalanced only at short distances by the two modified Bessel functions of the second kind appearing in the numerator, which diverge logarithmically whenever $\sqrt{2 E c}|\mathbf{u}|=0$ and $/$ or $\sqrt{2 E c}|\mathbf{v}|=0$. The total result of these opposite effects in the expression of the averaged second moment will be presented in Section VI after performing the explicit computation of the amplitudes of Eqs. (44-48).

To conclude this Section, let us give some concrete values of the involved parameters. First of all, let us estimate the values of $L$, for which the two polymer system is in the regime (101). Using the approximations made in Eq. (98), we may conclude that, if the relation (101) is satisfied, the length $L$ needs such that $L \gg 2 a$, i. e. $L$ is at least of the order of hundred molecular lengths or more: $L>100 a$. Moreover, it is possible to give a rough estimation of the maximum distance of the end points, after which the two polymers are too far from each other to allow a relevant contribution to the winding angle due to repulsive interactions. Using Eq. (94), in fact, it turns out that the repulsive interactions are relevant only in the range of distances:

$$
|\mathbf{u}| \ll \sqrt{\frac{L a}{2}} \sim l_{r e p}
$$


Finally, the situation opposite to condition (101) is not realistic, because it leads to the constraint $L \ll 2 a$. This would corresponds to the case of a polymer which is shorter than the size of the molecules composing it.

\section{CALCULATION OF THE AVERAGED SECOND MOMENT}

At this point we are ready to compute the quantities $N(E)$ and $D(E)$ of Eqs. (78) and (79). We start with $D(E)$. Using Eqs. (83), (87) and the splitting (84) of the propagator, one has at the zeroth order in $\lambda(E)$ :

$$
D^{(0)}(E)=\int d^{2} r_{0} \int d^{2} r_{1} G_{0}\left(E ; \mathbf{r}_{1}, \mathbf{r}_{0}\right)=\int d^{2} r_{0} \int d^{2} r_{1} \frac{c}{\pi} K_{0}\left(\sqrt{2 E c}\left|\mathbf{r}_{1}-\mathbf{r}_{0}\right|\right)
$$

After a shift of variables, the above equation gives:

$$
D^{(0)}(E)=S \int d^{2} r_{1} \frac{c}{\pi} K_{0}\left(\sqrt{2 E c}\left|\mathbf{r}_{1}\right|\right)
$$

where $S=\int d^{2} r_{0}$ is the total surface of the system in the two dimensional space, which is transverse to the $t$-axis. Using the identity

$$
\int d^{2} r_{1} \frac{c}{\pi} K_{0}\left(\sqrt{2 E c}\left|\mathbf{r}_{1}\right|\right)=\frac{1}{E}
$$

one finds:

$$
D^{(0)}(E)=S / E
$$

This expression of $D^{(0)}(E)$ has the following interpretation: We are performing here an average of the second moment with respect to all possible initial and final positions of the endpoints of the polymers and $D(E)$ counts the number of these configurations. The component $D^{(0)}(E)$ of $D(E)$ depends only on the free propagator $G_{0}(E ; \mathbf{x}, \mathbf{y})$, which is translational invariant in the sense discussed after Eq. (162). This invariance explains why the number of configurations grows proportionally to the surface $S$. The reason is that, for each configuration of the polymers, one can obtain other equivalently probable configurations by the symmetric translation of their ends on the surface $S$ at the initial and final instants. Let us now apply to $D^{(0)}(E)$ an inverse Laplace transform, in order to go back to the $L$-space. After a simple calculation we obtain:

$$
D^{(0)}(L)=S
$$


i. e. $D^{(0)}(L)$ does not depend on $L$.

The next and last contribution to $D(E)$ is given by:

$$
D^{(1)}(E)=\int d^{2} r_{0} d^{2} r_{1} G_{1}\left(E ; \mathbf{r}_{1}, \mathbf{r}_{0}\right)=\int d^{2} r_{0} d^{2} r_{1} \frac{c}{\pi} \lambda(E) K_{0}\left(\sqrt{2 E c}\left|\mathbf{r}_{1}\right|\right) K_{0}\left(\sqrt{2 E c}\left|\mathbf{r}_{0}\right|\right)
$$

Exploiting Eq. (107) to integrate out the variables $\mathbf{r}_{0}$ and $\mathbf{r}_{1}$, we get:

$$
D^{(1)}(E)=\frac{\pi}{c} \lambda(E) E^{-2}
$$

We remark that the above contribution to $D(E)$ vanishes in the limit $v_{0}=0$. This could be expected due to the fact that $D^{(1)}(E)$ collects all contributions coming from the repulsive interactions. These interactions break explicitly the translational invariance of the free part of the action and, as a consequence, $D^{(1)}(E)$ is no longer proportional to the surface $S$ as $D^{(0)}(E)$. Unfortunately, it is not easy to compute the inverse Laplace transform of $D^{(1)}(E)$ without making some approximation. To this purpose, we assume that the repulsive interactions are weak, i. e. $v_{0} \ll 1$, and that the value of $L$ is large. In this case, since we are in the domain of small $E^{\prime}$ 's, it is possible to expand $D^{(1)}(E)$ up to the second order in $v_{0}$ as follows:

$$
D^{(1)}(E) \sim \frac{\pi}{c}\left(\frac{c}{\pi} E^{-2} v_{0}-\left(\frac{c}{\pi} v_{0}\right)^{2} E^{-2} \log (\sqrt{2 E c} a)\right)
$$

In order to obtain the above equation we have used both Eqs. (63) and (97). The inverse Laplace transform of Eq. (112) gives:

$$
D^{(1)}(L) \sim\left[v_{0}-\frac{c}{\pi} v_{0}^{2}\left(\log (\sqrt{2 c} a)+\frac{C-1}{2}\right)\right] L+\frac{c}{2 \pi} v_{0}^{2} L \log L
$$

where $C \sim 0.577215664$ is the Euler constant.

Putting Eqs. (108) and (111) together, we obtain:

$$
D(E)=D^{(0)}(E)+D^{(1)}(E)=S E^{-1}+\frac{\pi}{c} \lambda(E) E^{-2}
$$

This is an exact result. An approximated expression of $D(L)$ can be derived instead from Eqs. (109) and (113).

Now we turn to the derivation of $N(E)$. We start by computing order by order in $\lambda(E)$ the contributions to the quantities $K(E)$ and $I_{\omega}(E)$ of Eqs. (88) and (89) respectively. At the zeroth order we have for $K(E)$ :

$$
K^{(0)}(E)=\frac{c}{2 \pi^{2}} \int d^{2} x \mathbf{A}^{2}(\mathbf{x}) \int d^{2} r_{1} K_{0}\left(\sqrt{2 E c}\left|\mathbf{r}_{1}-\mathbf{x}\right|\right) \int d^{2} r_{0} K_{0}\left(\sqrt{2 E c}\left|\mathbf{r}_{0}-\mathbf{x}\right|\right)
$$


After performing an easy integrations over the coordinates $\mathbf{r}_{0}, \mathbf{r}_{1}$, one obtains:

$$
K^{(0)}(E)=\frac{1}{2 c} E^{-2} \int d^{2} x \mathbf{A}^{2}(\mathbf{x})
$$

The remaining integral with respect to the $\mathbf{x}$ coordinate is both ultraviolet and infrared divergent and needs to be regulated. We have already seen that the singularities in the ultraviolet domain may consistently be eliminated with the introduction of the small distance cut-off $a$. A large distance cut-off is instead motivated by the fact that the size of a real system is necessarily finite. Implicitly, we have already used this kind of infrared regularization in Eq. (106), where we have assumed that the total surface $S$ of the system in the directions which are transverse to the $t$-axis is finite. Supposing that the shape of $S$ is approximately a disk of radius $R$, so that $S \sim \pi R^{2}$, we may write:

$$
\int d^{2} x \mathbf{A}^{2}(\mathbf{x})=\frac{1}{2 \pi} \int_{a}^{R} \frac{d \rho}{\rho}
$$

Substituting Eq. (117) in Eq. (116), one obtains the following expression of $K^{(0)}(E)$ :

$$
K^{(0)}(E)=\frac{1}{8 \pi c} E^{-2} \log \left(\frac{S}{a^{2} \pi}\right)
$$

The inverse Laplace transform of $K^{(0)}(E)$ gives:

$$
K^{(0)}(L)=\frac{L}{8 \pi c} \log \left(\frac{S}{a^{2} \pi}\right)
$$

We have now to compute the quantities $I_{\omega}^{(0)}(E)$, with $\omega=1, \ldots, 4$. The expressions of the $I_{\omega}^{(0)}(E)$ 's may be obtained from Eqs. (81) and (45] 48), by substituting everywhere the propagator $G(E ; \mathbf{x}, \mathbf{y})$ with its free version $G_{0}(E ; \mathbf{x}, \mathbf{y})$. It is easy to show that:

$$
I_{\omega}^{(0)}(E)=0 \quad \text { for } \omega=1, \ldots, 4
$$

This vanishing, which is actually a double vanishing, is due to the fact that each of the $I_{\omega}^{(0)}(E)$ 's contains an integral of a total divergence together with an integral which is zero for symmetry reasons. For some values of $\omega$, like for instance when $\omega=3$, to isolate such integrals it is necessary to perform some integrations by parts. This is allowed because the $I_{\omega}^{(0)}(E)$ 's are not affected by divergences, contrarily to $K(E)$.

As an example, we work out explicitly the case of $I_{1}^{(0)}(E)$. The first vanishing integral is the following:

$$
\int d^{2} r_{0} \nabla_{\mathbf{y}}^{j} G_{0}\left(E ; \mathbf{r}_{0}, \mathbf{y}\right)=\frac{c}{\pi} \int d^{2} r_{0} \nabla_{\mathbf{y}}^{j} K_{0}\left(\sqrt{2 E c}\left|\mathbf{r}_{0}-\mathbf{y}\right|\right)
$$


This is of course zero due to symmetry reasons. The second vanishing integral in $I_{1}^{(0)}(E)$ is of the form:

$$
I=\int d^{2} x \int d^{2} r_{1} A_{i}(\mathbf{x}) G_{0}\left(E ; \mathbf{r}_{1}, \mathbf{x}\right) \nabla_{\mathbf{x}}^{i} G_{0}(E ; \mathbf{y}, \mathbf{x})
$$

After performing the integration over $\mathbf{r}_{1}$ with the help of a shift of variables and of Eq. (107), we have, apart from a proportionality factor:

$$
I \propto \int d^{2} x A_{i}(\mathbf{x}) \nabla_{\mathbf{x}}^{i} G_{0}(E ; \mathbf{y}, \mathbf{x})
$$

Since $A_{i}(\mathbf{x})$ is a divergenceless vector potential, i. e. $\nabla_{\mathbf{x}}^{i} A_{i}(\mathbf{x})=0, I$ can be rewritten as the integral of a total divergence:

$$
I=\frac{c}{\pi} \int d^{2} x \nabla_{\mathbf{x}}^{i}\left(A_{i}(\mathbf{x}) K_{0}(\sqrt{2 E c}|\mathbf{y}-\mathbf{x}|)\right)
$$

Clearly, the left hand side of the above equation is zero. This fact can be also checked passing to the Fourier representation. Exploiting Eq. (65) and the formula

$$
A^{i}(\mathbf{x})=\frac{1}{(2 \pi)^{2} i} \int d^{2} \mathbf{p} \epsilon^{i j} \frac{p_{j}}{\mathbf{p}^{2}} e^{i \mathbf{p} \cdot \mathbf{x}}
$$

in Eq. (123), one obtains:

$$
I=-\frac{1}{(2 \pi)^{2}} \int d^{2} \mathbf{p} \frac{\epsilon^{i j} p_{i} p_{j}}{\left(\mathbf{p}^{2}+2 E c\right) \mathbf{p}^{2}}
$$

Thus $I=0$ because $\epsilon^{i j} p_{i} p_{j}=0$. In an analogous way one shows that also $I_{2}^{(0)}, I_{3}^{(0)}$ and $I_{4}^{(0)}$ are identically equal to zero.

We are now ready to compute the contributions to $N(E)$, which are linear in $\lambda(E)$. First of all, we treat the term $K^{(1)}(E)$, which is given by:

$$
K^{(1)}(E)=\frac{1}{2 c} \int d^{2} x \int d^{2} r_{0} \int d^{2} r_{1} \mathbf{A}^{2}(\mathbf{x})\left[G_{1}\left(E ; \mathbf{r}_{1}, \mathbf{x}\right) G_{0}\left(E ; \mathbf{x}, \mathbf{r}_{0}\right)+G_{0}\left(E ; \mathbf{r}_{1}, \mathbf{x}\right) G_{1}\left(E ; \mathbf{x}, \mathbf{r}_{0}\right)\right]
$$

The integrations over $\mathbf{r}_{0}$ and $\mathbf{r}_{1}$ may be easily performed using Eq. (107) and give as a result a factor which is proportional to $E^{-2}$. After that, only the following integral in $\mathbf{x}$ remains to be done:

$$
\int d^{2} x \mathbf{A}^{2}(\mathbf{x}) K_{0}(\sqrt{2 E c}|\mathbf{x}|) \equiv \frac{1}{(2 \pi)^{2}} \int_{|\mathbf{x}| \geq a} d^{2} x \frac{1}{|\mathbf{x}|^{2}} K_{0}(\sqrt{2 E c}|\mathbf{x}|)
$$

Here the ultraviolet divergence, which is present in the left hand side, has been regulated in the usual way with the introduction of the short distances cut-off $a$. Infrared divergences 
are absent. Going to polar coordinates, the right hand side of the above equation becomes:

$$
\frac{1}{(2 \pi)^{2}} \int_{|\mathbf{x}| \geq a} d^{2} x \frac{1}{|\mathbf{x}|^{2}} K_{0}(\sqrt{2 E c}|\mathbf{x}|)=\frac{1}{2 \pi} \int_{a}^{+\infty} d \rho \frac{K_{0}(\sqrt{2 E c} \rho)}{\rho}
$$

Putting everything together, one arrives at the final result:

$$
K^{(1)}(E)=\frac{1}{2 \pi c} E^{-2} \lambda(E) \int_{a}^{+\infty} d \rho \frac{K_{0}(\sqrt{2 E c} \rho)}{\rho}
$$

If the quantity $\sqrt{2 E c} a$ is small, it is possible to derive the following asymptotic expression of $K^{(1)}(E)$ :

$$
K^{(1)}(E) \sim \frac{1}{4 \pi c} E^{-2} \lambda(E) \log ^{2}(\sqrt{2 E c} a)
$$

To go from Eq. (130) to Eq. (131), we have used the asymptotic formula:

$$
\int_{a}^{+\infty} d \rho \frac{K_{0}(\sqrt{2 E c} \rho)}{\rho} \sim \frac{1}{2} \log ^{2}(\sqrt{2 E c} a)
$$

which is valid for small values of $\sqrt{2 E c} a$. We see from Eqs. (130) and (132) that the presence of ultraviolet divergences, together with the needed regularization, has modified the naive form of $K^{(1)}(E)$ as a function of the pseudo-energy $E$ given in Eq. (90). The modification consists in the appearance of the factor $\int_{a}^{+\infty} \frac{d \rho}{\rho} K_{0}(\sqrt{2 E c} \rho)$, which exhibits a square logarithmic singularity in the limit $\sqrt{2 E c} a=0$.

The inverse Laplace transformed of $K^{(1)}(E)$ can be derived only making some approximation. As in the case of $D^{(1)}(E)$, we will work in the double limit, in which $v_{0}$ is very small and $L$ is very large. After a few calculations we obtain:

$$
\begin{aligned}
K^{(1)}(L) \sim & \frac{v_{0}}{4 \pi^{2}}\left\{\frac{1}{4} \int_{0}^{L} d s[\log (L-s)+C](\log s+C)+\right. \\
& \left.\frac{1}{4} L \log ^{2}\left(2 c a^{2}\right)+\frac{1}{2} \log \left(2 c a^{2}\right)[(C-1) L-L \log L]\right\}
\end{aligned}
$$

At this point we have to compute the expressions of the $I_{\omega}^{(1)}(E)$ 's, $\omega=1, \ldots, 4$. It is possible to show that these contributions vanish identically, i. e.:

$$
I_{\omega}^{(1)}(E)=0 \quad \text { for } \omega=1, \ldots, 4
$$

The motivations of this vanishing are similar to the motivations for which there are no contributions at the zeroth order: All terms which appear in the quantities $I_{\omega}^{(1)}(E)$ contain at least one integral of a total divergence or one integral, which is zero for dimensional 
reasons. As in the case of the $I_{\omega}^{(0)}(E)$ 's, there are some values of $\omega$ for which it is necessary to perform an integration by parts in order to isolate these vanishing integrals. Once again, this is allowed because the $I_{\omega}^{(1)}(E)$ 's do not contain divergences.

At the next order in $\lambda(E)$, we have the last contribution to $K(E)$ :

$$
K^{(2)}(E)=\frac{1}{2 c} \int d^{2} x \int d^{2} r_{1} \int d^{2} r_{0} \mathbf{A}^{2}(\mathbf{x}) G_{1}\left(E ; \mathbf{r}_{1}, \mathbf{x}\right) G_{1}\left(E ; \mathbf{x}, \mathbf{r}_{0}\right)
$$

After performing the integrations in $\mathbf{r}_{1}$ and $\mathbf{r}_{0}$ with the help of Eq. (107), Eq. (135) becomes:

$$
K^{(2)}(E)=\frac{\pi}{c} \lambda^{2}(E) E^{-2} \int d^{2} x \mathbf{A}^{2}(\mathbf{x})\left(K_{0}(\sqrt{2 E c}|\mathbf{x}|)\right)^{2}
$$

The integral in $\mathbf{x}$ is divergent and needs a regularization. Going to polar coordinates, we obtain the result:

$$
K^{(2)}(E) \equiv \frac{\pi}{c} \lambda^{2}(E) E^{-2} \int_{a}^{+\infty} \frac{d \rho}{\rho}\left(K_{0}(\sqrt{2 E c} \rho)\right)^{2}
$$

Also in this case, we note that the presence of the regularization modifies the dependence of $K^{(2)}(E)$ on the pseudo-energy $E$ with respect to the naive formula of Eq. (90). The correction consists in the factor $\int_{a}^{+\infty} \frac{d \rho}{\rho}\left(K_{0}(\sqrt{2 E c} \rho)\right)^{2}$. In the limit $\sqrt{2 E c} a=0$, this factor diverges as powers of $\log (\sqrt{2 E c} a)$.

To conclude the analysis of the contribution to $N(E)$ at the second order in $\lambda(E)$, we show that the $I_{\omega}^{(2)}(E)$ 's are identically equal to zero. As a matter of fact, it is easy to verify that for $\omega=1,2,4$ each $I_{\omega}^{(2)}(E)$ contains terms of the kind:

$$
B(\mathbf{x})=A_{i}(\mathbf{x}) \nabla_{\mathbf{x}}^{i} K_{0}(\sqrt{2 E c}|\mathbf{x}|)
$$

These terms vanish identically because of the following identity:

$$
\nabla_{\mathbf{x}}^{i} K_{0}(\sqrt{2 E c}|\mathbf{x}|)=\frac{1}{\sqrt{2 E c}} \frac{x^{i}}{|\mathbf{x}|^{2}} \frac{\partial K_{0}(\sqrt{2 E c}|\mathbf{x}|)}{\partial|\mathbf{x}|}
$$

Substituting Eq. (139) in Eq. (138) and using the explicit expression of the vector potential $A_{i}(\mathbf{x})$ of Eq. (17), we get:

$$
B(\mathbf{x})=\frac{1}{2 \pi \sqrt{2 E c}} \frac{\epsilon_{j i} x^{i} x^{j}}{|\mathbf{x}|^{4}} \frac{\partial K_{0}(\sqrt{2 E c}|\mathbf{x}|)}{\partial|\mathbf{x}|}
$$

Clearly, the right hand side of the above equation is zero because $\epsilon_{j i} x^{i} x^{j}=0$. If $\omega=3$, instead, the vanishing function $B(\mathbf{x})$ of Eq. (138) may be isolated in the expression of $I_{3}^{(2)}(E)=0$ only after an integration by parts. 
Finally, at the third order in $\lambda(E)$ we have only the quantities $I_{\omega}^{(3)}(E)$ 's, since $K(E)$ has at most quadratic powers of $\lambda(E)$. It is easy to realize that:

$$
\sum_{\omega=1}^{4} I_{\omega}^{(3)}(E)=0
$$

because the following relations hold [46]:

$$
I_{1}^{(3)}(E)=-I_{2}^{(3)}(E)=I_{3}^{(3)}(E)=-I_{4}^{(3)}(E)
$$

As a consequence of Eq. (141), it is clear that there are no contributions to $N(E)$ at this order.

Using Eqs. (118), (130) and (137), we arrive at the final result for $N(E)$ :

$$
\begin{aligned}
N(E) & =\frac{1}{4 \pi c} E^{-2} \log \left(\frac{S}{a^{2} \pi}\right)+\frac{1}{\pi c} \lambda(E) E^{-2} \int_{a}^{+\infty} \frac{d \rho}{\rho} K_{0}(\sqrt{2 E c} \rho) \\
& +\frac{2 \pi}{c} \lambda^{2}(E) E^{-2} \int_{a}^{+\infty} \frac{d \rho}{\rho}\left(K_{0}(\sqrt{2 E c} \rho)\right)^{2}
\end{aligned}
$$

We can now insert in the formula of the second moment of Eq. (77) the functions $D(E)$ and $N(E)$ given in Eqs. (114) and (143) respectively. The outcome is:

$$
\left\langle m^{2}\right\rangle(E)=\frac{E^{-1}\left[\frac{1}{4 \pi c} \log \left(\frac{S}{a^{2} \pi}\right)+\lambda(E) \frac{\int_{a}^{+\infty} \frac{d \rho}{\rho} K_{0}(\sqrt{2 E c} \rho)}{\pi c}+\lambda^{2}(E) \frac{2 \pi}{c} \int_{a}^{+\infty} \frac{d \rho}{\rho}\left(K_{0}(\sqrt{2 E c} \rho)\right)^{2}\right]}{S+\frac{\pi}{c} \lambda(E) E^{-1}}
$$

In the $L$-space, the already mentioned difficulties with the computation of the inverse Laplace transform of $D(E)$ and $N(E)$ allow an analytical result only in the double limit of weak coupling constant $v_{0}$ and of large values of $L$. At the first order in $v_{0}$, the expression of $\left\langle m^{2}\right\rangle$ reads as follows:

$$
\left\langle m^{2}\right\rangle=\frac{\frac{L}{8 \pi c} \log \left(\frac{S}{a^{2} \pi}\right)+K^{(1)}(L)}{S+v_{0} L}
$$

where $K^{(1)}(L)$ has been given in Eq. (133).

So far, we have considered the averaged second moment of Eq. (26), corresponding to the case in which the polymer ends are not fixed. In the energy representation, we have seen that this version of the second moment can be exactly computed. To conclude this Section, we would like to show that it is possible to provide also an exact expression of the second moment $\left\langle m^{2}\right\rangle_{\mathbf{r}_{1}, \mathbf{r}_{0}}$ in the $L-$ space and with fixed polymer ends up to an inverse Laplace transform of the propagator given in Eq. (64). The starting point is the exact formula of 
the second moment $\left\langle m^{2}\right\rangle \mathbf{r}_{1}, \mathbf{r}_{0}(E)$ of Eq. (49)). All the ingredients of this formula are defined in Eqs. (26), (38)-(39) and (411)-(48). Looking at Eq. (49), it is clear that:

$$
N\left(E ; \mathbf{r}_{1}, \mathbf{r}_{0}\right)=2 K\left(\mathbf{r}_{1}, \mathbf{r}_{0}\right)-\sum_{\omega=1}^{4} I_{\omega}\left(\mathbf{r}_{1}, \mathbf{r}_{0}\right)
$$

and

$$
D\left(E ; \mathbf{r}_{1}, \mathbf{r}_{0}\right)=G\left(E ; \mathbf{r}_{1}, \mathbf{r}_{0}\right)
$$

Let us note that the functions $I_{\omega}\left(\mathbf{r}_{1}, \mathbf{r}_{0}\right)$ are all equal up to integrations by parts, which can shift the differential operators $\nabla_{\mathbf{x}}$ and $\nabla_{\mathbf{y}}$ in Eqs. (444)-(48). This fact will be used in order to simplify the expression of the inverse Laplace transformed of $N\left(E ; \mathbf{r}_{1}, \mathbf{r}_{0}\right)$ in the $L$-space. To compute the inverse Laplace transforms of both $N\left(E ; \mathbf{r}_{1}, \mathbf{r}_{0}\right)$ and $D\left(E ; \mathbf{r}_{1}, \mathbf{r}_{0}\right)$, we use the following property of the inverse Laplace transform of the product of two functions $f(E)$ and $g(E)$ :

$$
\mathcal{L}^{-1}(f(E) g(E))=\int_{0}^{L} d s f(L-s) g(s)
$$

Applying Eq. (148) to evaluate the inverse Laplace transforms of $K(E)$ and of the $I_{\omega}\left(\mathbf{r}_{1}, \mathbf{r}_{0}\right)$ in Eqs. (146) and (147), we obtain after some calculations:

$$
\begin{aligned}
N\left(L ; \mathbf{r}_{1}, \mathbf{r}_{0}\right) & =\frac{2}{c} \int d^{2} x \mathbf{A}^{2}(\mathbf{x}) \int_{0}^{L} d s G\left(L-s ; \mathbf{r}_{1}, \mathbf{x}\right) G\left(s ; \mathbf{x}, \mathbf{r}_{0}\right) \\
& -\frac{2}{c^{2}} \int d^{2} x \int d^{2} y \int_{0}^{L} G\left(L-s ; \mathbf{x}, \mathbf{r}_{1}\right) \\
& \times \int_{0}^{s} d s^{\prime} \partial_{x}^{i} \partial_{y}^{j} G\left(s-s^{\prime} ; \mathbf{y}, \mathbf{x}\right) G\left(s^{\prime} ; \mathbf{r}_{0}, \mathbf{y}\right) A_{i}(\mathbf{x}) A_{j}(\mathbf{y}) \\
D\left(L ; \mathbf{r}_{1}, \mathbf{r}_{0}\right) & =G\left(L ; \mathbf{r}_{1}, \mathbf{r}_{0}\right)
\end{aligned}
$$

The second term in the right hand side of Eq. (149) is the contribution given by the functions $I_{\omega}\left(\mathbf{r}_{1}, \mathbf{r}_{0}\right), \omega=1, \ldots, 4$, while the first term comes from $K\left(\mathbf{r}_{1}, \mathbf{r}_{0}\right)$. Remembering the definition (21) of the second moment in terms of $N\left(L ; \mathbf{r}_{1}, \mathbf{r}_{0}\right)$ and $D\left(L ; \mathbf{r}_{1}, \mathbf{r}_{0}\right)$, we get:

$$
\begin{gathered}
\left\langle m^{2}\right\rangle \mathbf{r}_{1}, \mathbf{r}_{0}=\left[G\left(L ; \mathbf{r}_{1}, \mathbf{r}_{0}\right)\right]^{-1}\left[\frac{2}{c} \int d^{2} x \mathbf{A}^{2}(\mathbf{x}) \int_{0}^{L} d s G\left(L-s ; \mathbf{r}_{1}, \mathbf{x}\right) G\left(s ; \mathbf{x}, \mathbf{r}_{0}\right)\right. \\
\left.-\frac{2}{c^{2}} \int d^{2} x \int d^{2} y \int_{0}^{L} d s G\left(L-s ; \mathbf{x}, \mathbf{r}_{1}\right) \int_{0}^{s} d s^{\prime} \partial_{x}^{i} \partial_{y}^{j} G\left(s-s^{\prime} ; \mathbf{y}, \mathbf{x}\right) G\left(s^{\prime} ; \mathbf{r}_{0}, \mathbf{y}\right) A_{i}(\mathbf{x}) A_{j}(\mathbf{y})\right]
\end{gathered}
$$

If we knew how to compute the propagator $G(L ; \mathbf{x}, \mathbf{y})$ starting from its Laplace transformed (64), we could evaluate explicitly the expression of the second moment in the $L$-space. Unfortunately, it is too complicated to perform the inverse Laplace transform of the propagator $G(E ; \mathbf{x}, \mathbf{y})$. Due to this technical difficulty, Eq. (1511) is only formal. Progress can be 
made however in the limit $v_{0}=0$, in which the propagator is given by the Green function $G_{0}(E ; \mathbf{x}, \mathbf{y})$ of Eq. (62). This will be done in the next Section.

\section{THE CASE OF IDEAL POLYMERS}

In order to allow the comparison with previous results, this Section is dedicated to the case of ideal chains in which $v_{0}=0$. First of all, we discuss the formula of the averaged second moment derived in the previous Section, Eq. (145). In the limit $v_{0}=0$, Eq. (145) becomes:

$$
\left\langle m^{2}\right\rangle_{0}=\frac{L}{8 \pi c S} \log \left(\frac{S}{a^{2} \pi}\right)
$$

The presence of a geometrical factor like the surface $S$ of the system in the expression of $\left\langle m^{2}\right\rangle_{0}$ has been already related to the translational symmetry of Eqs. (75) and (76). Assuming that this surface has approximately the shape of a disc of radius $R$, we can put $S=\pi R^{2}$ as in Eq. (117). Eq. (152) predicts that the average degree of entanglement scales as follows with respect to the distance $R$ :

$$
\left\langle m^{2}\right\rangle_{0} \propto \frac{\log R}{R^{2}}
$$

The meaning of Eq. (153) is the following. We remember that the averaged second moment $\left\langle m^{2}\right\rangle_{0}$ describes the entanglement of two closed polymers whose ends on the surfaces at $t=0$ and $t=L$ are not fixed. In this way, the polymers are allowed to move freely and it is natural to suppose that, the bigger will be the volume $S L$ in which the polymers fluctuate, the bigger will be also the average distance between them. Thus, if the surface $S$ increases its area, the probability of entanglement must decrease. The exact law of this decreasing is given by Eq. (153).

On the other side, one would expect that the probability of getting entangled is higher for long polymers than for short polymers. Eq. (152) gives a result which is in agreement with the above expectation, because the second moment $\left\langle m^{2}\right\rangle_{0}$ scales as follows with respect to the parameters $L$ and $c$, which determine the polymer length:

$$
\left\langle m^{2}\right\rangle_{0} \propto \frac{L}{c}
$$

In particular, one can show that the total length of a polymer increases proportionally to $L$ and it is inversely proportional to the square root of $c$ [36]. Accordingly, we see from Eq. (154) that $\left\langle m^{2}\right\rangle_{0}$ increases proportionally to $L$ and inversely proportional to $c$. 
At this point we wish to study the second moment $\left\langle m^{2}\right\rangle_{0}, \mathbf{r}_{1}, \mathbf{r}_{0}$ of polymers with fixed endpoints. The subscript 0 has been added to the symbol of the second moment to remember that we are working in the limit $v_{0}=0$. Since we are dealing with ideal polymers, we have to substitute everywhere in Eq. (151) the full propagator $G(L ; \mathbf{x}, \mathbf{y})$ with the free one. The result of this operation is:

$$
\begin{array}{r}
\left\langle m^{2}\right\rangle_{0, \mathbf{r}_{1}, \mathbf{r}_{0}}=\left[G_{0}\left(L ; \mathbf{r}_{1}, \mathbf{r}_{0}\right)\right]^{-1}\left[\frac{2}{c} \int d^{2} x \mathbf{A}^{2}(\mathbf{x}) \int_{0}^{L} d s G_{0}\left(L-s ; \mathbf{r}_{1}, \mathbf{x}\right) G_{0}\left(s ; \mathbf{x}, \mathbf{r}_{0}\right)-\right. \\
\left.\frac{2}{c^{2}} \int d^{2} x \int d^{2} y \int_{0}^{L} d s G_{0}\left(L-s ; \mathbf{x}, \mathbf{r}_{1}\right) \int_{0}^{s} d s^{\prime} \partial_{x}^{i} \partial_{y}^{j} G_{0}\left(s-s^{\prime} ; \mathbf{y}, \mathbf{x}\right) G_{0}\left(s^{\prime} ; \mathbf{r}_{0}, \mathbf{y}\right) A_{i}(\mathbf{x}) A_{j}(\mathbf{y})\right]
\end{array}
$$

We notice that, as it could be expected, Eq. (155) coincides with the expression obtained in [8] for the second moment of one polymer winding up around an infinitely long straight wire lying along the $z$-axis. Luckily, the propagator $G_{0}\left(L ; \mathbf{r}_{1}, \mathbf{r}_{0}\right)$ can be explicitly constructed upon computing the inverse Laplace transform of the propagator $G_{0}\left(E ; \mathbf{r}_{1} ; \mathbf{r}_{0}\right)$ of Eq. (62):

$$
G_{0}(L ; \mathbf{x}, \mathbf{y})=\frac{c}{2 \pi L} e^{\frac{c}{2 L}|\mathbf{x}-\mathbf{y}|^{2}}
$$

It is easy to check that the second term in the right hand side of Eq. (155), which is associated with the contributions coming from the $I_{\omega}\left(\mathbf{r}_{1}, \mathbf{r}_{0}\right)$ 's, does not grow with increasing values of $L$. As a matter of fact, after a rescaling of variables, the numerator of this term gives:

$$
\begin{aligned}
& \frac{2}{c^{2}} \int d^{2} x \int d^{2} y \int_{0}^{L} d s G_{0}\left(L-s ; \mathbf{x}, \mathbf{r}_{1}\right) \int_{0}^{s} d s^{\prime} \partial_{x}^{i} \partial_{y}^{j} G_{0}\left(s-s^{\prime} ; \mathbf{y}, \mathbf{x}\right) G_{0}\left(s^{\prime} ; \mathbf{r}_{0}, \mathbf{y}\right) A_{i}(\mathbf{x}) A_{j}(\mathbf{y})= \\
& \frac{c}{4 \pi^{3} L} \int d^{2} x^{\prime} \int d^{2} y^{\prime} \int_{0}^{1} d t \frac{1}{1-t} e^{-\frac{c}{2(1-t)}\left|\mathbf{x}^{\prime}-\frac{\mathbf{r}_{1}}{L}\right|^{2}} \int_{0}^{t} d t^{\prime} \frac{1}{t-t^{\prime}}\left[\frac{\partial^{2}}{\partial_{x_{i}^{\prime}} \partial_{y_{j}^{\prime}}} e^{-\frac{c}{2\left(t-t^{\prime}\right)}\left|\mathbf{x}^{\prime}-\mathbf{y}^{\prime}\right|^{2}}\right] \\
& \frac{1}{t^{\prime}} e^{-\frac{c}{2\left(t^{\prime}\right)}\left|\mathbf{y}^{\prime}-\frac{\mathbf{r}_{0}}{L}\right|^{2}}
\end{aligned}
$$

In the limit $L \longrightarrow \infty$, the quantity in the right hand side of the above equation scales as $A L^{-1}$, where $A$ is a constant. Moreover, the propagator (156), which is in the denominator, scales as $L^{-1}$. Thus, the ratio between the right hand side of Eq. (157) and the propagator (156) does not depend on $L$. This completes the proof of our statement.

As a consequence of this statement, as far as the scaling of $\left\langle m^{2}\right\rangle_{0}, \mathbf{r}_{1}, \mathbf{r}_{0}$ for large values of $L$ is concerned, it is possible to make the following approximation:

$$
\left\langle m^{2}\right\rangle_{0, \mathbf{r}_{1}, \mathbf{r}_{0}} \sim \frac{2}{c}\left[G_{0}\left(L ; \mathbf{r}_{1}, \mathbf{r}_{0}\right)\right]^{-1} \int d^{2} x \mathbf{A}^{2}(\mathbf{x}) \int_{0}^{L} d s G_{0}\left(L-s ; \mathbf{r}_{1}, \mathbf{x}\right) G_{0}\left(s ; \mathbf{x}, \mathbf{r}_{0}\right)
$$


Unfortunately, despite of the fact that we are treating ideal polymers, the integral in $d^{2} x$ appearing in the above equation is still complicated and requires some approximation to be evaluated analytically. We will apply to this purpose the strategy used in Ref. [8] to compute the second moment of three dimensional polymers, adapting it to our two-dimensional case. First of all, let us note that the integral in (158) is ultraviolet divergent. However, the infrared divergences which appeared in the energy representation are absent. This is due to the behavior of the propagator $G_{0}(L ; \mathbf{x}, \mathbf{y})$, which is much milder at infinity than the behavior of the Green function $G_{0}(E ; \mathbf{x}, \mathbf{y})$. To regulate the singularities at small distances, we proceed as usual by introducing the cut-off $a$. After a rescaling of all variables similar to that of Eq. (157), we get:

$$
\begin{gathered}
\left\langle m^{2}\right\rangle_{0, \mathbf{r}_{1}, \mathbf{r}_{0}} \sim \frac{2}{c}\left[G_{0}\left(L ; \mathbf{r}_{1}, \mathbf{r}_{0}\right)\right]^{-1} \times \\
\int_{\left|x^{\prime}\right| \geq \frac{a \sqrt{c}}{\sqrt{L}}} \frac{d^{2} x^{\prime}}{L} \frac{1}{\mathbf{x}^{\prime 2}} \int_{0}^{1} \frac{d s^{\prime}}{s^{\prime}\left(1-s^{\prime}\right)}\left(\frac{c}{2 \pi}\right)^{2} e^{-\frac{1}{2\left(1-s^{\prime}\right)}\left|\mathbf{x}^{\prime}-\mathbf{r}_{1} \sqrt{\frac{c}{L}}\right|^{2}} e^{-\frac{1}{2 s^{\prime}}\left|\mathbf{x}^{\prime}-\mathbf{r}_{0} \sqrt{\frac{c}{L}}\right|^{2}}
\end{gathered}
$$

To go further, following [8], we assume that the relevant contribution to the integral in $d^{2} x^{\prime}$ comes from a narrow region around the singularity in $x^{\prime}=0$. Thus, we may put

$$
\begin{aligned}
& \int_{\left|x^{\prime}\right| \geq \frac{a \sqrt{c}}{\sqrt{L}}} \frac{d^{2} x^{\prime}}{\mathbf{x}^{\prime 2}} e^{-\frac{1}{2\left(1-s^{\prime}\right)}\left|\mathbf{x}^{\prime}-\mathbf{r}_{1} \sqrt{\frac{c}{L}}\right|^{2}} e^{-\frac{1}{2\left(1-s^{\prime}\right)}\left|\mathbf{x}^{\prime}-\mathbf{r}_{0} \sqrt{\frac{c}{L}}\right|^{2}}
\end{aligned}
$$

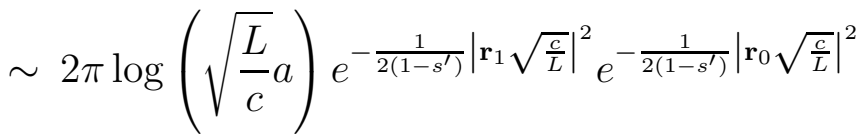

After making the above crude approximation, we obtain:

$$
\begin{aligned}
& \left\langle m^{2}\right\rangle_{0, \mathbf{r}_{1}, \mathbf{r}_{0}} \sim \frac{c}{\pi L}\left[G_{0}\left(L ; \mathbf{r}_{1}, \mathbf{r}_{0}\right)\right]^{-1} \log \left(\sqrt{\frac{L}{c} a}\right) \int_{0}^{1} d s^{\prime}\left[\frac{1}{1-s^{\prime}} e^{-\frac{1}{2\left(1-s^{\prime}\right)} \mathbf{r}_{1}^{2} \frac{c}{L}} e^{-\frac{1}{2 s^{\prime}} \mathbf{r}_{0}^{2} \frac{c}{L}}+\right. \\
& \left.\frac{1}{s^{\prime}} e^{-\frac{1}{2\left(1-s^{\prime}\right)} \mathbf{r}^{2} \frac{c}{L}} e^{-\frac{1}{2 s^{\prime}} \mathbf{r}_{0}^{2} \frac{c}{L}}\right]
\end{aligned}
$$

In deriving the above equation we have used the simple relation $\frac{1}{s^{\prime}\left(1-s^{\prime}\right)}=\frac{1}{\left(1-s^{\prime}\right)}+\frac{1}{s^{\prime}}$. Let us now study the integral

$$
\tilde{I}=\int_{0}^{1} \frac{d s^{\prime}}{s^{\prime}} e^{-\frac{1}{2\left(1-s^{\prime}\right)} \mathbf{r}_{1}^{2} \frac{c}{L}} e^{-\frac{1}{2 s^{\prime}} \mathbf{r}_{0}^{2} \frac{c}{L}}
$$

The other integral in $d s^{\prime}$ appearing in (161) can be treated in the same way after the change of variables $1-s^{\prime}=t$. It is not to allowed to take in the right hand side of Eq. (162) the limit $L \longrightarrow \infty$ because in this way the integral will not be convergent due to the singularity in $s^{\prime}=0$. For this reason, we split the domain of integration as follows:

$$
\tilde{I}=\int_{0}^{u} \frac{d s^{\prime}}{s^{\prime}} e^{-\frac{1}{2\left(1-s^{\prime}\right)} \mathbf{r}_{1}^{2} \frac{c}{L}} e^{-\frac{1}{2 s^{\prime}} \mathbf{r}_{0}^{2} \frac{c}{L}}
$$




$$
+\int_{u}^{1} \frac{d s^{\prime}}{s^{\prime}} e^{-\frac{1}{2\left(1-s^{\prime}\right)} \mathbf{r}_{1}^{2} \frac{c}{L}} e^{-\frac{1}{2 s^{\prime}} \mathbf{r}_{0}^{2} \frac{c}{L}}
$$

where $0<u<1$. Clearly, the second integral converges after performing the limit $L \longrightarrow \infty$ in the integrand and gives:

$$
\int_{u}^{1} \frac{d s^{\prime}}{s^{\prime}}=\log \frac{1}{u}
$$

The first integral instead diverges logarithmically with growing values of $L$. However, now it is possible to expand the exponential $e^{-\frac{1}{2\left(1-s^{\prime}\right)} \mathbf{r}_{1}^{2} \frac{c}{L}}$ in powers of its argument, because the singularity in $s^{\prime}=1$ lies outside the interval $[0, u]$. Keeping only the leading order term with respect to $L$, we get:

$$
\tilde{I} \sim-\operatorname{Ei}\left(-\frac{u \mathbf{r}_{0}^{2} c}{2 L}\right)-\log u
$$

where $\operatorname{Ei}(z)$ is the exponential-integral function. When $L$ is large, this function may be approximated as follows: $\operatorname{Ei}(z) \sim \log (-z)$ and, as a consequence:

$$
\tilde{I} \sim-\log \left(\frac{\mathbf{r}_{0}^{2} c}{2 L}\right)
$$

The second integral which we have left in Eq. (161) gives the same result. Putting everything together in the expression of the second moment of Eq. (161), we obtain the final result:

$$
\left\langle m^{2}\right\rangle_{0, \mathbf{r}_{1}, \mathbf{r}_{0}} \sim-2 \log \left(\sqrt{\frac{L}{c}} a\right) \log \left(\frac{\mathbf{r}_{1}^{2} \mathbf{r}_{0}^{2} c^{2}}{4 L^{2}}\right) \sim 2(\log L)^{2}
$$

This is exactly the behavior of the second moment derived in Ref. [8].

\section{CONCLUSIONS}

In this article we have studied the entanglement of two directed polymers from a nonperturbative point of view. Our formulas of the second moment, a quantity which measures the degree of entanglement of the two polymers, take into account the repulsive forces acting on the segments of the polymers and are exact. The averaged second moment defined in Eq. (18), a version of the second moment corresponding to the situation in which the end points of the polymers are free to move, has been computed in Eq. (144) as a function of the chemical potential $E$ conjugated to the distance $L$ between the end points in the $t$-direction. The case of free ends is relevant in the treatment of nematic polymers and polymers in a nematic solvent [21]. Let us note that also the expression of the second moment without any 
averaging and in the $L$ space can be computed. This has been done in Eq. (151). However, this equation is explicit only up to the inverse Laplace transform of the propagator (64), which is too hard to be obtained in closed form.

Eq. (144) shows that the averaged second moment is of the form $\left\langle m^{2}\right\rangle(E)=E^{-1} f(E)$. The overall factor $E^{-1}$ coincides with the scaling power law of two ideal polymers. The correction $f(E)$ to this fundamental behavior due to the repulsive interactions is a complicated function of $E$, whose analysis would require numerical methods. Nevertheless, it is possible to identify a dominance of the repulsive interactions in the domain of parameters in which the condition $\sqrt{2 E c} a \sim 0$ is satisfied. This corresponds roughly speaking to the situation in which polymers are very long. In this region, the scaling laws with respect to the energy $E$ of the numerator and denominator appearing in the right hand side of Eq. (144) are corrected by factors which are $\log$ arithmic powers of $\log (\sqrt{2 E c} a)$, see for instance Eq. (132).

One advantage of our approach is that it is easy to separate within the expression of the second moment the contributions of purely entropic origin which are typical of free polymers from the contributions coming from the presence of the $\delta$-function potential in the polymer action. This is essentially due to the splitting (25) of the propagator $G(E ; \mathbf{u}, \mathbf{v})$ appearing in the amplitudes (444 48) . The component $G_{0}(E ; \mathbf{u}, \mathbf{v})$ of the propagator coincides with the propagator of ideal polymers, while the component $G_{1}(E ; \mathbf{u}, \mathbf{v})$ takes into account the effects of the interactions. Thanks to the splitting (95)), it has been possible to study the way in which the repulsive forces affect the average degree of entanglement of the two polymers. This has been done in Section $\mathbf{D}$ Our results are in agreement with the intuition. The precise law with which the effects of the repulsive forces on the entanglement decrease when the distance between the trajectories increases is given by Eq. (95)). In Section $\nabla$ it has been discussed also the strong coupling limit, which should be taken to recover the limit of excluded volume interactions. In our exact approach, it is not difficult to consider the case in which the coupling constant $v_{0}$ is large. For instance, the component $G_{1}(E ; \mathbf{u}, \mathbf{v})$ of the propagator, which is responsible of the effects due to the repulsive interactions, has been given in the strong coupling limit in Eq. (99). Studying the form of this component assuming that polymers are very long, it has been argued that, at strong coupling, the major contributions to the winding angle coming from the repulsive interactions occur when the trajectories are very near to each other. Many other qualitative and quantitative characteristics of the behavior of the two polymer system under consideration have been presented in 


\section{Section $\mathrm{V}$}

The case of ideal polymers, in which $v_{0}=0$, has been discussed at the end of Section VII in order to make comparison with previous works. The scaling of the averaged second moment for large values of $L$ obtained in Eq. (154) is in agreement with the results of [7], if one takes into account the fact that, after the averaging procedure of Eq. (18) and the infrared regularization of Eqs. (106) and (117). one is effectively treating a system of polymers confined in a cylinder of finite volume $S L$. In Section VII we have evaluated the second moment, always of two ideal polymers, using the approach of Ref. [8]. The outcome of this calculation, namely the scaling behavior of $\left\langle m^{2}\right\rangle_{0}, \mathbf{r}_{1}, \mathbf{r}_{0}$ at the leading order in $L$, is reported in Eq. (167). This result is in agreement with the square logarithmic behavior obtained in [8], but not with the logarithmic behavior predicted in [7]. However, this discrepancy can be expected due to the fact that, in Section VII], we have assumed, following Ref. [8], that the most relevant contribution to the second moment coming from the integral in Eq. (159) is concentrated in a narrow region near the singularity in $x^{\prime}=0$. This clashes with the assumptions of Ref. [7], in which instead it is argued that the main increase in the winding angle does not occur when the polymer trajectories are near, but rather when they are far one from the other. Finally, there is also an apparent discrepancy between the linear scaling with respect to $L$ of the averaged second moment $\left\langle m^{2}\right\rangle_{0}$ and the square logarithmic scaling

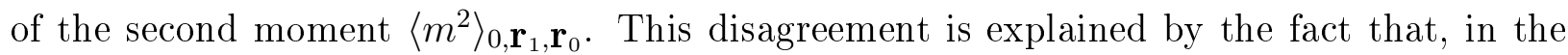
first case, the ends of the polymers are free to fluctuate, while in the second case they are fixed. It is therefore licit to expect that two polymers with free ends are more likely to entangle than two polymers whose ends are constrained.

Concluding, we would like to discuss possible further developments of this work, together with some problems which are still left open. First of all, the number of entangling polymers has been limited to two. To go beyond this restriction, one should explore the possibility of replacing the external vector potential $A_{i}(\mathbf{x})$ of Eq. (7) with Chern-Simons fields. Abelian Chern-Simons field theories have been already successfully applied in order to impose topological constraints to the trajectories of an arbitrary number of closed polymer rings in [37]. We hope to extend those results also to the case of directed polymers in a forthcoming publication. Of course, if the polymer trajectories are open, the constraints among them are no longer of topological nature as in [37], so that the application of Chern-Simons field theory to directed polymers should be considered with some care. 
We have also not made any attempt to introduce in the treatment of polymer entanglement more sophisticated constraints than those which can be imposed with the help of the winding angle. This is in effect still an unsolved problem, despite the fact that two powerful and strategies have been proposed for its solution [38, 39, 40]. In the first approach, pioneered independently by Kleinert, Kholodenko and one of the authors [19, 38, 39]. the constraints are expressed via the Wilson loop amplitudes of non-abelian Chern-Simons field theories. Some progresses toward a concrete realization of this program in polymer physics have been made in Refs. [41, 42]. In the second approach, developed by Nechaev and coworkers, see [40] and references therein. polymer trajectories are mapped on a complex plane with punctures. The link invariants necessary to impose the constraints are then constructed using the properties of conformal maps.

Another possible development is the treatment of attractive interactions, in which the strength $v_{0}$ in Eq. (2) takes negative values. In this case, the $\delta$-function potential admits a bound state [32] and the propagator of Eq. (64) develops a singularity, in which $\lambda(E)=\infty$, at the energy corresponding to this bound state. It would be extremely interesting to investigate how these facts affect the polymers' entanglement. Another issue which deserves attention is that of hairpin turns. Hairpins are important in nematic solvents [21] and can be included with the help of field theories [43]. We note also that in our formalism it is also possible to study the entanglement of polymers in confined geometries. For example, values of $E$ which are near to $a^{-1}\left(E \leq a^{-1}\right)$ correspond roughly speaking to the situation in which polymers fluctuate in a quasi two-dimensional environment, in which the height in the $t$-direction is of the order of a few molecular sizes.

Finally, an open problem, which has not be discussed here because we were mainly interested in the second moment. is the derivation of the full partition function $\mathcal{G}_{\lambda}\left(E ; \mathbf{r}_{1}, \mathbf{r}_{0}\right)$ of Eq. (19). As anticipated in the Introduction, it is not an easy task to compute $\mathcal{G}_{\lambda}\left(E ; \mathbf{r}_{1}, \mathbf{r}_{0}\right)$ because the repulsive potential of Eq. (2) is not central. We note however that the expression of $\mathcal{G}_{\lambda}\left(E ; \mathbf{r}_{1}, \mathbf{r}_{0}\right)$ coincides with the Green function of a spin $1 / 2$ Aharonov-Bohm problem in the imaginary time formulation of quantum mechanics. This Green function has been already derived in [29] using sophisticated techniques developed in Refs. [27, 32], which bypass all the difficulties of dealing with a non-central potential. Thus, in principle, the expression of the partition function $\mathcal{G}_{\lambda}\left(E ; \mathbf{r}_{1}, \mathbf{r}_{0}\right)$ is known. Unfortunately, some of the consistency conditions imposed on the parameters in Ref. [29] seem to be incompatible with the requirements of 
our physical problem, as noted in Section III. For these reasons, the computation of the full partition function $\mathcal{G}_{\lambda}\left(E ; \mathbf{r}_{1}, \mathbf{r}_{0}\right)$ is still a problem which needs further investigations. Luckily, the knowledge of the partition function is not necessary if one is interested to study the excluded volume interactions, which arise in the strong coupling limit. In fact, in this case it is possible to apply a powerful method due to Kleinert [33, 34, 35]. This method turns the weak coupling expansion into a strong coupling expansion which is convergent for large values of $v_{0}$ and is able to accommodate also the anomalous dimensions of quantum field theories. The convergence of this strong coupling expansion is mostly very fast, so that only a few coefficients of the weak coupling expansion must be known, see Refs. [19, 35] for more details. These coefficients can be easily computed starting from the well known partition function of the Aharonov-Bohm problem without the insertion of the $\delta$-function potential [19] and treating this potential as a small perturbation assuming that the value of $v_{0}$ is small. The application of Kleinert's method in order to complete the brief analysis of the strong coupling limit made in this paper is work in progress.

[1] S. A. Wasserman and N. R. Cozzarelli, Science 232 (1986), 951; S. D. Levene, C. Donahue, T. C. Boles and N. R. Cozzarelli, Biophys. Jour. 69 (1995), 1036; F. Livolant and Y. Bouligand, J. Phys. (Paris) 47 (1986), 1813; R. Podgornik, D. C. Rau and V. A. Parsegian, Macromolecules 22 (1989), 1780.

[2] S. Chandrasekhar, B. K. Sadashiva and K. A. Suresh, Pramana 9 (1977), 471; Nguyen Huu Tinh, H. Gasparoux and C. Destrade, Mol. Cryst. Liq. Cryst. 68 (1981), 101; T. K. Attwood, J. E. Lyndon and F. Jones, Liq. Crys. 1 (1986), 499.

[3] C. Bélisle, Ann. Prob. 17 (1989), 1377.

[4] J. W. Pitman and M. Yor, Ann. Prob. 14 (1986), 733.

[5] A. Comtet, J. Desbois and C. Monthus, Jour. Stat. Phys. 73 (1993), 433.

[6] A. Grosberg and S. Nechaev, Polymer Topology, Advances in Polymer Science 106, (Springer Verlag, 1993), 1.

[7] B. Drossel and M. Kardar, in Theoretical and mathematical models in polymer research, A. Grosberg (Editor), (Academic Press, 1998), 187; cond-mat/9610019; Phys. Rev. E53 (1996), 5861. 
[8] F. Tanaka, Prog. Theor. Phys. 68 (1982), 148.

[9] D. R. Nelson, in Observation, prediction and simulation of phase transitions in complex fluids, M. Baus, L. F. Rull and J. P. Ryckaert (Eds.), (Kluwer, The Netherlands, 1995).

[10] J. D. Moroz and R. D. Kamien, Nucl. Phys. B506 [FS] (1997), 695.

[11] A. L. Kholodenko and T. A. Vilgis, Phys. Rep. 298 (1998), 251.

[12] F. Ferrari and I. Lazzizzera, Phys. Lett. B 444 (1998) 167; F. Ferrari, H. Kleinert and I. Lazzizzera, Eur. Phys. J. B18 (2000), 645.

[13] M. Otto, J. Phys. A: Math. Gen. 34 (2001), 2539; Topological interactions in systems of mutually interlinked polymer rings, cond-mat/0402104.

[14] M. G. Brereton and S. Shah, J. Phys. A: Math. Gen. 15 (1982), 989.

[15] S. Edwards, Proc. Phys. Soc. 91 (1967), 513; J. Phys. A1 (1968), 15.

[16] F. Spitzer, Trans. Am. Math. Soc. 87 (1958), 187.

[17] S. Prager and H. L. Frisch, J. Chem. Phys. 46 (1967), 1475.

[18] N. Saito and Y. Chen, J. Chem. Phys. 59 (1973), 3701.

[19] H. Kleinert, Path Integrals in Quantum Mechanics, Statistics, Polymer Physics, and Financial Markets, (World Scientific Publishing, 3nd Ed., Singapore, 2003).

[20] M. Kardar and Y.-C. Zhang, Phys. Rev. Lett. 58 (1987). 2087; M. Kardar, G. Parisi and Y.-C. Zhang, Phys. Rev. Lett. 56 (1986), 889.

[21] R. D. Kamien, P. Le Doussal and D. R. Nelson, Phys. Rev. A 45 (12) (1992), 8727.

[22] K. Iwata and T. Kimura, J. Chem. Phys. 74 (1981), 2039.

[23] H. Bethe and R. Peierls, Proc. Roy. Soc. (London) 148A (1935), 146; E. Fermi, Ricerca Scientifica 7 (1936), 13, [English translation: E. Fermi, Collected Papers Vol. 1. Italy 19211938 (University of Chicago Press, Chicago, 1962)].

[24] F. Berezin and L. Faddeev, Sov. Math. Dokl. 2 (1961), 372.

[25] S. Albeverio, F. Geztesy, R. Hoeg-Krohn and H. Holden, Solvable models in quantum mechanics (Springer Verlag, Berlin, 1988); S. Albeverio, Z. Brzeźniak andL. Dąbrowski, Jour. Phys. A 27 (1994), 4933.

[26] P. Gerbert, Phys. Rev. D 40 (1989), 1346.

[27] C. R. Hagen, Phys. Rev. Lett. 64 (1990), 503; Int. Jour. Mod. Phys. A4 (1991), 3119.

[28] C. Grosche, Phys. Rev. Lett. 71 (1993), 1; Ann. Phys. 3 (1994), 283.

[29] D. K. Park, Jour. Math. Phys. 36 (10) (1995), 5453. 
[30] R. P. Feynman and A. R. Hibbs, Quantum Mechanics and Path Integrals, (McGraw-Hill, New York, 1965).

[31] L. S. Schulman, Techniques and Applications of Path Integrals, (Wiley, New York, 1981).

[32] R. Jackiw, Delta-function potential in two- and three-dimensional quantum mechanics, in M. A. Bég Memorial Volume, A. Ali and P. Hoodbhoy (Eds.), (Worlds Scientific, Singapore, 1991).

[33] H. Kleinert, Phys. Rev. D57 (1998), 2264.

[34] H. Kleinert, Phys. Lett. B434 (1998), 74, [arXiv: cond-mat/9801167].

[35] H. Kleinert and V. Schulte-Frohlinde, Critical properties of $\Phi^{4}$-Theories, World Scientific, Singapore 2001.

[36] F. Ferrari, Phys. Lett. A323 (2004), 351, cond-mat/0401104.

[37] F. Ferrari, H. Kleinert and I. Lazzizzera, Int. J. Mod. Phys. B14 (2000) 3881 [arXiv: cond-mat/0005300].

[38] H. Kleinert, Pfadintegrale in Quantenmechanik, Statistik un Polymerphysik, Spektrum Akademischer Verlag, Heidelberg, 1st Edition 1993, 850p., ISBN 3-86025-613-0.

[39] A. Kholodenko, Trends in Chem. Physics Vol. 3, Research Trends Inc. Trivandrum, India (1994), pp. 63-94.

[40] S. Nechaev and R. Voituriez, Conformal Geometry and Invariants of 3-strand Brownian Braids, [arXiv: hep-th/0407008].

[41] F. Ferrari, Chern-Simons Field Theories with Non-semisimple Gauge Group of Symmetry Jour. Math. Phys. 44 (1) (2003), 138, hep-th/0210100.

[42] F. Ferrari, Topological field theories with non-semisimple gauge group of symmetry and engineering of topological invariants, published in Trends in Field Theory Research, O. Kovras (Ed.), Nova Science Publishers (2004).

[43] J. Cardy, Jour. Phys. A 16 (1983), L355.

[44] For details, see Ref. [12], where a similar calculation has been done in the case of closed polymers.

[45] Throughout this Section we will use a notation in which the vectors $\mathbf{x}, \mathbf{y}$ appearing in the definition of the propagator of Eq. (64) are replaced by the vectors $\mathbf{u}, \mathbf{v}$. This is to avoid confusions with the notation of Eqs. (44, 48), where the same pair of vectors $\mathbf{x}, \mathbf{y}$ has been exploited to denote dummy integration variables.

[46] Let us stress the fact that each of the $I_{\omega}^{(3)}(E)$ 's is separately equal to zero, because these 
quantities contain terms of the kind given in Eq. (138). 